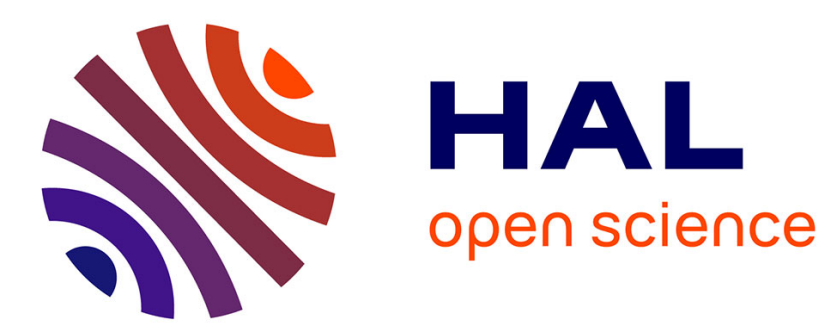

\title{
Reduced-order model for the BGK equation based on POD and optimal transport
}

\author{
Florian Bernard, Angelo Iollo, Sébastien Riffaud
}

\section{To cite this version:}

Florian Bernard, Angelo Iollo, Sébastien Riffaud. Reduced-order model for the BGK equation based on POD and optimal transport. Journal of Computational Physics, 2018, 373, pp.545-570. 10.1016/j.jcp.2018.07.001 . hal-01943540

\section{HAL Id: hal-01943540 \\ https://hal.science/hal-01943540}

Submitted on 3 Dec 2018

HAL is a multi-disciplinary open access archive for the deposit and dissemination of scientific research documents, whether they are published or not. The documents may come from teaching and research institutions in France or abroad, or from public or private research centers.
L'archive ouverte pluridisciplinaire HAL, est destinée au dépôt et à la diffusion de documents scientifiques de niveau recherche, publiés ou non, émanant des établissements d'enseignement et de recherche français ou étrangers, des laboratoires publics ou privés. 


\title{
Reduced-order model for the BGK equation based on POD and optimal transport
}

\author{
Florian Bernard ${ }^{\mathrm{a}, \mathrm{b}}$, Angelo Iollo ${ }^{\mathrm{a}, \mathrm{b}}$, Sébastien Riffaud ${ }^{\mathrm{a}, \mathrm{b}, *}$ \\ ${ }^{a} I M B$, UMR 5251, Univ. Bordeaux, 33400 Talence, France \\ ${ }^{b}$ INRIA Bordeaux Sud-Ouest, Team MEMPHIS, 33400 Talence, France
}

\begin{abstract}
We present a reduced-order approximation of the BGK equation leading to fast and accurate computations. The BGK model describes the dynamics of a gas flow in both hydrodynamic and rarefied regimes. The particles of the gas are represented by a density distribution function depending on physical space, velocity space and time. In this work, the density distribution function is approximated in the velocity space by a small number of basis functions computed offline. In the offline phase, the BGK equation is sampled in order to collect information on the density distribution function. To complete this sampling, optimal transport is used to add new information by interpolating the samples of the density distribution function. Finally, the basis functions are built by Proper Orthogonal Decomposition. During the online phase, the offline knowledge is used to compute approximations of the density distribution function at low cost. To do so, the BGK equation is projected onto the basis functions, leading to a system of partial differential equations. The system obtained is hyperbolic by construction and is solved by an IMEX Runge-Kutta scheme in time and a finite-volume scheme in space. To improve the accuracy, the reduced-oder model is modified to conserve mass, momentum and energy of the gas. Numerical illustrations of 1D and 2D flows are given. In particular, we investigate the reconstruction and the prediction of shock waves, boundary layers and vortices. The results demonstrate the accuracy of the reduced-order model and the significant reduction of the computational cost.
\end{abstract}

Keywords: Reduced-order model, Proper Orthogonal Decomposition, Rarefied flows, Optimal transportation

\section{Introduction}

We present a model describing a gas flow in both hydrodynamic and rarefied regimes. These regimes are characterized by the Knudsen number

$$
K n=\frac{\lambda}{L}
$$

${ }^{*}$ Corresponding author: sebastien.riffaud@u-bordeaux.fr sebastien.riffaud@inria.fr 
where $\lambda$ is the mean free path of the particles and $L$ is the characteristic length of the problem. When the Knudsen number is low (roughly $K n<10^{-2}$ ) the collisions between the gas particles are preponderant compared to the free transport of the particles and the regime is hydrodynamic. Conversely, the rarefied regime corresponds to $10^{-2}<K n$.

In the high-fidelity model, the dynamics of the gas flow is described by the Boltzmann equation

$$
\frac{\partial f}{\partial t}(\mathbf{x}, \xi, t)+\xi \cdot \nabla_{\mathbf{x}} f(\mathbf{x}, \xi, t)=Q(f, f)
$$

where $f$ is the gas density distribution function at point $\mathbf{x} \in \mathbb{R}^{3}$, velocity $\xi \in \mathbb{R}^{3}$ and time $t \in \mathbb{R}$. $Q$ is the collision operator, approximated here by the BGK operator [1]. The computational cost of this model can be prohibitive due to the high number of dimensions of the grid (e.g. 3 in space +3 in velocity +1 in time).

For this reason, we develop a stable, accurate and efficient reduced-order model to compute approximations of the density distribution function $f$ at low cost with respect to the high-fidelity model. To this end, several approaches were developed in the literature: Proper Orthogonal Decomposition [2, 3, 4, 5, 6, 4, 8, 9, 10, 11, 12, Proper Generalised Decomposition [13], Empirical Interpolation Method [14, 15]. In this work, we adopt an approach based on Proper Orthogonal Decomposition and Galerkin method. In this approach, the gas density distribution function $f$ is approximated in velocity space by $N_{\text {pod }}$ basis functions $\Phi_{n}$

$$
\widetilde{f}(\mathbf{x}, \xi, t)=\sum_{n=1}^{N_{\text {pod }}} a_{n}(\mathbf{x}, t) \Phi_{n}(\xi)
$$

The basis functions $\Phi_{n}$ are computed offline by Proper Orthogonal Decomposition [3] and the coefficients $a_{n}$ are computed online by the Galerkin method.

In the offline phase, the high-fidelity model is sampled to collect information on the density distribution function $f$ that we want to approximate. The sampling is based on two fundamental steps. Firstly, we collect samples of both the density distribution function $f$ and of the Maxwellian distribution function $M_{f}$, since $M_{f}$ will also be represented by the basis functions $\Phi_{n}$. Secondly, since the number of simulations may be limited due to the computational cost of the high-fidelity model, the samples of the distribution functions $f$ and $M_{f}$ are interpolated by optimal transport to compute additional samples, completing the sampling of the high-fidelity model. Finally, the basis functions $\Phi_{n}$ are built by POD to be the best representation in the least squares sense of the samples.

During the online phase, this offline knowledge is used to compute an approximation of the density distribution function $\widetilde{f}$ at low cost. In the Galerkin method, the Boltzmann equation (1) is projected onto the basis functions $\Phi_{n}$, leading to a system of partial differential equations that is shown to be hyperbolic by construction. The equations are then decoupled by a linear change of variables and the 
system is solved by a first-order IMEX Runge-Kutta scheme [16, 17, 18] in time and a first-order finite volume scheme [19] in space. Due to projection error, the approximation of the Maxwellian distribution function $\widetilde{M}_{f}$ does not necessarily preserve conservation leading to significant errors in $\tilde{f}$. We introduce a constraint projection to compute $\widetilde{M}_{f}$ in order to conserve the mass, the momentum and the energy of the gas that significantly improves accuracy.

These steps are detailed in the next sections. In section 2 , we introduce the high-fidelity model. Then section 3 presents in details the reduced-order model approximating the high-fidelity model. In section 4, we describe the boundary conditions and the numerical schemes used to discretize the reduced-order model. Finally, the last section 5 demonstrates the accuracy of the reduced-order model and the significant reduction of the computational cost.

\section{High-fidelity model}

We briefly introduce the high-fidelity model describing a gas flow in both hydrodynamic and rarefied regimes. The detailed model can be found in [20].

\subsection{Dimensionless BGK model}

The high-fidelity model uses the BGK operator [1] where the collision term $Q$ is linearised around the Maxwellian distribution function $M_{f}$ in the Boltzmann equation (1)

$$
\frac{\partial f}{\partial t}(\mathbf{x}, \xi, t)+\xi \cdot \nabla_{\mathbf{x}} f(\mathbf{x}, \xi, t)=\frac{M_{f}(\mathbf{x}, \xi, t)-f(\mathbf{x}, \xi, t)}{\tau(\mathbf{x}, t)}
$$

with $\mathbf{x}=(x, y, z)^{T} \in \mathbb{R}^{3}, \xi=\left(\xi_{u}, \xi_{v}, \xi_{w}\right)^{T} \in \mathbb{R}^{3}$ and $t \in \mathbb{R}$. For simplicity, we consider a monoatomic gas. In dimensionless form, the relaxation time $\tau$ is

$$
\tau^{-1}(\mathbf{x}, t)=\frac{\rho(\mathbf{x}, t) T^{1-\nu}(\mathbf{x}, t)}{K n}
$$

with $\nu \in \mathbb{R}$ the exponent of the viscosity law of the gas and $M_{f}$ the Maxwellian distribution function

$$
M_{f}(\mathbf{x}, \xi, t)=\frac{\rho(\mathbf{x}, t)}{(2 \pi T(\mathbf{x}, t))^{\frac{3}{2}}} \exp \left(-\frac{\|\xi-U(\mathbf{x}, t)\|^{2}}{2 T(\mathbf{x}, t)}\right)
$$

where $\rho(\mathbf{x}, t) \in \mathbb{R}$ is the density, $U(\mathbf{x}, t)=(u(\mathbf{x}, t), v(\mathbf{x}, t), w(\mathbf{x}, t))^{T} \in \mathbb{R}^{3}$ is the macroscopic velocity and $T(\mathbf{x}, t) \in \mathbb{R}$ is the temperature of the gas. The density, the momentum and the energy of the gas are given by the moments of the density distribution function $f$

$$
\int_{\mathbb{R}^{3}} f(\mathbf{x}, \xi, t)\left(\begin{array}{c}
1 \\
\xi \\
\frac{\|\xi\|^{2}}{2}
\end{array}\right) \mathrm{d} \xi=\left(\begin{array}{c}
\rho(\mathbf{x}, t) \\
\rho(\mathbf{x}, t) U(\mathbf{x}, t) \\
E(\mathbf{x}, t)
\end{array}\right)
$$


where $E(\mathbf{x}, t) \in \mathbb{R}$ is the energy of the gas. The temperature $T(\mathbf{x}, t)$ and the pressure $p(\mathbf{x}, t) \in \mathbb{R}$ of the gas are deduced from the moments

$$
T(\mathbf{x}, t)=\frac{2 E(\mathbf{x}, t)}{3 \rho(\mathbf{x}, t)}-\frac{\|U(\mathbf{x}, t)\|^{2}}{3} \quad \text { and } \quad p(\mathbf{x}, t)=\rho(\mathbf{x}, t) T(\mathbf{x}, t)
$$

\subsection{Discrete Maxwellian distribution function}

If $\langle.,\rangle_{\xi}$ denotes the discrete inner product used to approximate the continuous inner product, the Maxwellian distribution function $M_{f}$ does not necessarily verify the equation

$$
\left\langle M_{f}(\mathbf{x}, \xi, t), m(\xi)\right\rangle_{\xi}=\left(\begin{array}{c}
\rho(\mathbf{x}, t) \\
\rho(\mathbf{x}, t) U(\mathbf{x}, t) \\
E(\mathbf{x}, t)
\end{array}\right)
$$

where $m(\xi)=\left(1, \xi, \frac{\|\xi\|^{2}}{2}\right)^{T} \in \mathbb{R}^{5}$. For this reason, in the high-fidelity model, the discrete Maxwellian distribution function $\bar{M}_{f}$ is not computed from the formula (3). In [21, 22], it is proved that the discrete Maxwellian distribution function $\bar{M}_{f}$ can be express thanks to $\omega \in \mathbb{R}^{5}$

$$
\bar{M}_{f}(\mathbf{x}, \xi, t)=\exp (\omega(\mathbf{x}, t) \cdot m(\xi))
$$

To verify equation (5), $\omega$ must satisfy

$$
\left\langle\bar{M}_{f}(\mathbf{x}, \xi, t), m(\xi)\right\rangle_{\xi}=\left(\begin{array}{c}
\rho(\mathbf{x}, t) \\
\rho(\mathbf{x}, t) U(\mathbf{x}, t) \\
E(\mathbf{x}, t)
\end{array}\right)
$$

This nonlinear system is solved by the Newton-Raphson method at any point in space $\mathbf{x}$ and time $t$, see [20]. The equation (2) becomes after discretization in velocity space

$$
\frac{\partial f}{\partial t}(\mathbf{x}, \xi, t)+\xi \cdot \nabla_{\mathbf{x}} f(\mathbf{x}, \xi, t)=\frac{\bar{M}_{f}(\mathbf{x}, \xi, t)-f(\mathbf{x}, \xi, t)}{\tau(\mathbf{x}, t)}
$$

\subsection{Reduced BGK model}

To ensure equation (4), the velocity space has always 3 dimensions, even if the physical space has less dimensions. In $1 \mathrm{D}$ and 2D, the high-fidelity model uses two density distribution functions $\phi$ and $\psi$ as explained in [23] to reduce the number of dimensions in velocity space and to speed up the computations. In 1D, the density distribution function $f$ is replaced by

$$
\phi\left(x, \xi_{u}, t\right)=\int_{\mathbb{R}^{2}} f(x, \xi, t) \mathrm{d} \xi_{v} \mathrm{~d} \xi_{w} \quad \text { and } \quad \psi\left(x, \xi_{u}, t\right)=\int_{\mathbb{R}^{2}} \frac{\xi_{v}^{2}+\xi_{w}^{2}}{2} f(x, \xi, t) \mathrm{d} \xi_{v} \mathrm{~d} \xi_{w}
$$


By integrating in velocity space, equation (6) becomes

$$
\left\{\begin{array}{l}
\frac{\partial \phi}{\partial t}\left(x, \xi_{u}, t\right)+\xi_{u} \frac{\partial \phi}{\partial x}\left(x, \xi_{u}, t\right)=\frac{\bar{M}_{\phi}\left(x, \xi_{u}, t\right)-\phi\left(x, \xi_{u}, t\right)}{\tau(x, t)} \\
\frac{\partial \psi}{\partial t}\left(x, \xi_{u}, t\right)+\xi_{u} \frac{\partial \psi}{\partial x}\left(x, \xi_{u}, t\right)=\frac{\bar{M}_{\psi}\left(x, \xi_{u}, t\right)-\psi\left(x, \xi_{u}, t\right)}{\tau(x, t)}
\end{array}\right.
$$

and the equation (4) is replaced by

$$
\int_{\mathbb{R}} \phi\left(x, \xi_{u}, t\right)\left(\begin{array}{c}
1 \\
\xi_{u} \\
\frac{\xi_{u}^{2}}{2}
\end{array}\right) \mathrm{d} \xi_{u}+\int_{\mathbb{R}} \psi\left(x, \xi_{u}, t\right)\left(\begin{array}{l}
0 \\
0 \\
1
\end{array}\right) \mathrm{d} \xi_{u}=\left(\begin{array}{c}
\rho(x, t) \\
\rho(x, t) u(x, t) \\
E(x, t)
\end{array}\right)
$$

In $2 \mathrm{D}$, the new density distribution functions are

$$
\phi\left(\mathbf{x}, \xi_{2}, t\right)=\int_{\mathbb{R}} f(\mathbf{x}, \xi, t) \mathrm{d} \xi_{w} \quad \text { and } \quad \psi\left(\mathbf{x}, \xi_{2}, t\right)=\int_{\mathbb{R}} \frac{\xi_{w}^{2}}{2} f(\mathbf{x}, \xi, t) \mathrm{d} \xi_{w}
$$

with $\mathbf{x}=(x, y)^{T} \in \mathbb{R}^{2}$ and $\xi_{2}=\left(\xi_{u}, \xi_{v}\right)^{T} \in \mathbb{R}^{2}$. They verify

$$
\left\{\begin{array}{l}
\frac{\partial \phi}{\partial t}\left(\mathbf{x}, \xi_{2}, t\right)+\xi_{2} \cdot \nabla_{\mathbf{x}} \phi\left(\mathbf{x}, \xi_{2}, t\right)=\frac{\bar{M}_{\phi}\left(\mathbf{x}, \xi_{2}, t\right)-\phi\left(\mathbf{x}, \xi_{2}, t\right)}{\tau(\mathbf{x}, t)} \\
\frac{\partial \psi}{\partial t}\left(\mathbf{x}, \xi_{2}, t\right)+\xi_{2} \cdot \nabla_{\mathbf{x}} \psi\left(\mathbf{x}, \xi_{2}, t\right)=\frac{\bar{M}_{\psi}\left(\mathbf{x}, \xi_{2}, t\right)-\psi\left(\mathbf{x}, \xi_{2}, t\right)}{\tau(\mathbf{x}, t)}
\end{array}\right.
$$

and the moments are given by

$$
\int_{\mathbb{R}^{2}} \phi\left(\mathbf{x}, \xi_{2}, t\right)\left(\begin{array}{c}
1 \\
\xi_{u} \\
\xi_{v} \\
\frac{\left\|\xi_{2}\right\|^{2}}{2}
\end{array}\right) \mathrm{d} \xi_{2}+\int_{\mathbb{R}^{2}} \psi\left(\mathbf{x}, \xi_{2}, t\right)\left(\begin{array}{l}
0 \\
0 \\
0 \\
1
\end{array}\right) \mathrm{d} \xi_{2}=\left(\begin{array}{c}
\rho(\mathbf{x}, t) \\
\rho(\mathbf{x}, t) u(\mathbf{x}, t) \\
\rho(\mathbf{x}, t) v(\mathbf{x}, t) \\
E(\mathbf{x}, t)
\end{array}\right)
$$

The discrete Maxwellians $\bar{M}_{\phi}$ and $\bar{M}_{\psi}$ are computed to verify the equation (8) (resp. (10) ) in 1D (resp. 2D), see [20].

\section{Reduced-order model}

In the reduced-order model the density distribution function $f$ is approximated in the velocity space by $N_{\text {pod }}$ basis functions $\Phi_{n}$

$$
\widetilde{f}(\mathbf{x}, \xi, t)=\sum_{n=1}^{N_{\text {pod }}} a_{n}(\mathbf{x}, t) \Phi_{n}(\xi)
$$

in order to reduce the number of degrees of freedom. The basis functions $\Phi_{n}$ are built offline by Proper Orthogonal Decomposition and are orthonormal by construction. 
In $1 \mathrm{D}$ and in $2 \mathrm{D}$, the density distribution functions $\phi$ and $\psi$ of the high-fidelity model are modelled in the same way by

$$
\begin{aligned}
& \widetilde{\phi}\left(x, \xi_{u}, t\right)=\sum_{n=1}^{N_{\text {pod }}^{\phi}} a_{n}^{\phi}(x, t) \Phi_{n}^{\phi}\left(\xi_{u}\right) \text { and } \widetilde{\psi}\left(x, \xi_{u}, t\right)=\sum_{n=1}^{N_{\text {pod }}^{\psi}} a_{n}^{\psi}(x, t) \Phi_{n}^{\psi}\left(\xi_{u}\right) \quad \text { in 1D } \\
& \widetilde{\phi}\left(\mathbf{x}, \xi_{2}, t\right)=\sum_{n=1}^{N_{\text {pod }}^{\phi}} a_{n}^{\phi}(\mathbf{x}, t) \Phi_{n}^{\phi}\left(\xi_{2}\right) \text { and } \widetilde{\psi}\left(\mathbf{x}, \xi_{2}, t\right)=\sum_{n=1}^{N_{p o d}^{\psi}} a_{n}^{\psi}(\mathbf{x}, t) \Phi_{n}^{\psi}\left(\xi_{2}\right) \quad \text { in 2D }
\end{aligned}
$$

In the following we will detail the model for $\widetilde{f}$ (the method for $\widetilde{\phi}$ and $\widetilde{\psi}$ is similar).

\subsection{Basis functions}

In the offline phase, the high-fidelity model is sampled to collect information on the solution. The basis functions are computed by POD as explained in the following section.

\subsubsection{Snapshot database}

The sampling of the high-fidelity model provides a database $S$ containing $N_{\text {snaps }}$ snapshots $s_{l}$ of the density distribution function $f$

$$
S=\left\{s_{1}, s_{2}, \ldots, s_{N_{\text {snaps }}}\right\}
$$

where a snapshot $s_{l}$ is the density distribution function $f$ at a point $\mathbf{x}$ and time $t$, e.g., $s_{l}(\xi)=f\left(\mathbf{x}_{i(l), j(l), k(l)}, \xi, t_{p(l)}\right)$. In Figure 1, we show examples of snapshots in $1 \mathrm{D}$ and $2 \mathrm{D}$ where we take a low Knudsen number $\left(K n \approx 10^{-5}\right)$. In this case, the snapshots are close to a Maxwellian distribution function.
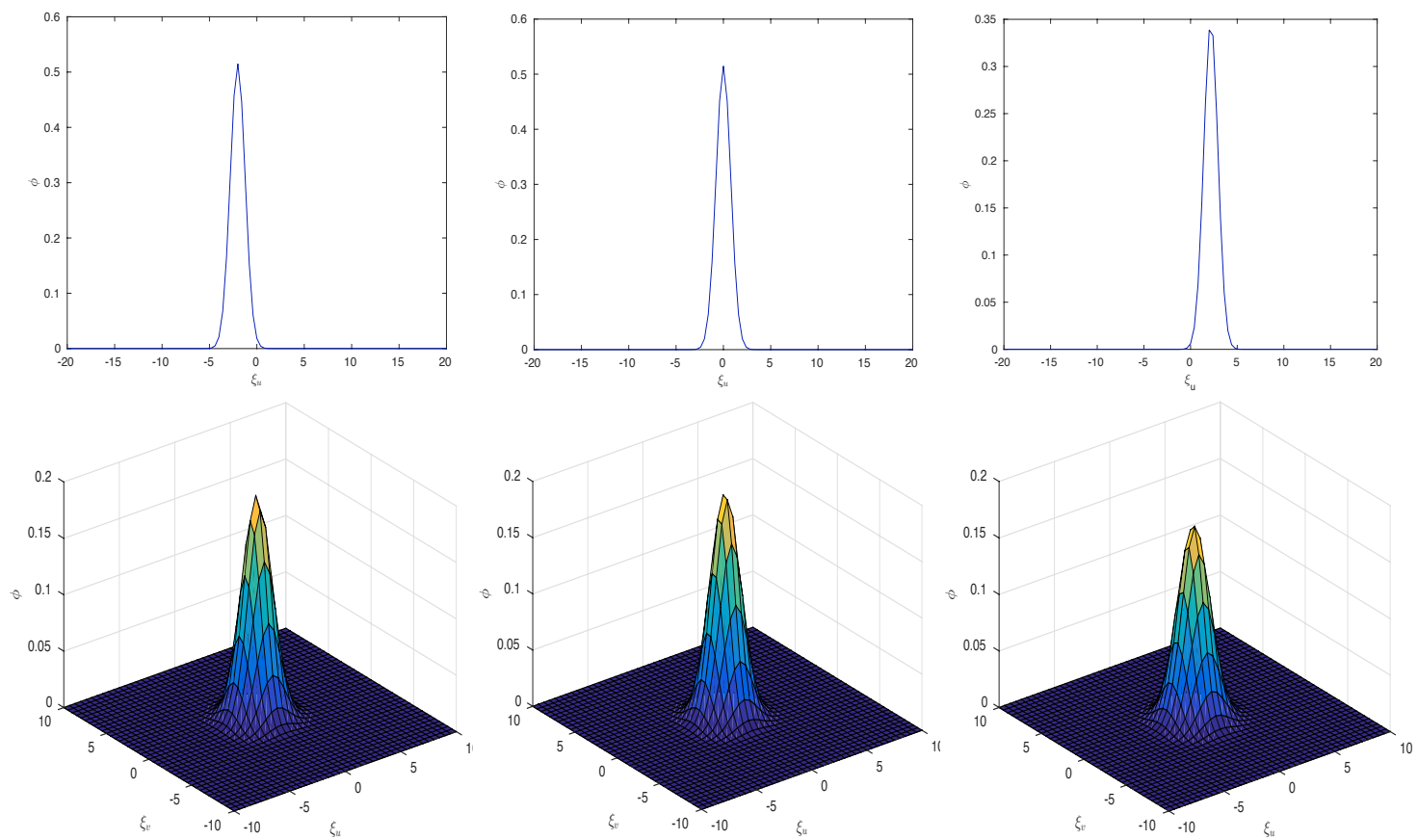

Figure 1: Examples of snapshots of $\phi$ in 1D (top) and in 2D (bottom) randomly choosen. 


\subsubsection{High-fidelity snapshots.}

The snapshots $s$ are provided by the high-fidelity model and contains snapshots of the density distribution function $f$ taken at any points in space and every time steps $t_{p}$

$$
S=\left\{f\left(\mathbf{x}_{i(m), j(m), k(m)}, \xi, t_{p}\right)\right\}_{\substack{1 \leq m \leq N_{\mathbf{x}} \\ 1 \leq p \leq N_{t}}}
$$

where we use 3D indexing for the space grid (i.e. $\left.\Omega_{\mathbf{x}}=\bigcup_{m=1}^{N_{\mathbf{x}}} \mathbf{x}_{i(m), j(m), k(m)}\right)$. In this way, the snapshots are uniformly distributed to represent the density distribution function $f$.

The database $S$ contains also snapshots of the discrete Maxwellian distribution function $\bar{M}_{f}$, since $M_{f}$ will be represented by the basis functions $\Phi_{n}$ in section 3.2 .2

$$
S=\left\{f\left(\mathbf{x}_{i(m), j(m), k(m)}, \xi, t_{p}\right)\right\}_{\substack{1 \leq m \leq N_{\mathbf{x}} \\ 1 \leq p \leq N_{t}}} \bigcup\left\{\bar{M}_{f}\left(\mathbf{x}_{i(m), j(m), k(m)}, \xi, t_{p}\right)\right\}_{\substack{1 \leq m \leq N_{\mathbf{x}} \\ 1 \leq p \leq N_{t}}}
$$

The results of this choice are presented in section 5.1 .2 .

\subsubsection{Low-fidelity snapshots.}

Due to the computational cost of the high-fidelity model, the number of simulations may be limited. To enrich the database $S$ with new snapshots, optimal transport [24] can be used to interpolate the distribution functions $f$ and $\bar{M}_{f}$ in velocity space. These additional low-fidelity snapshots are computed by the entropic regularization of optimal transport [25] which allows fast computations.

Let $\gamma>0$, the discrete entropy-regularized Wasserstein distance $\mathcal{W}_{\gamma}$ between two distributions $\mu_{1}, \mu_{2} \in \mathbb{R}_{+}^{N_{\xi}}$ is defined by

$$
\mathcal{W}_{\gamma}^{2}\left(\mu_{1}, \mu_{2}\right)=\min _{\pi \in \Pi\left(\mu_{1}, \mu_{2}\right)}\langle C, \pi\rangle_{F}-\gamma H(\pi)
$$

where $\pi \in \mathcal{M}_{N_{\xi}}\left(\mathbb{R}_{+}\right)$is the transportation plan (i.e. $\pi_{i, j}$ is the mass displaced from $\xi_{i}$ to $\left.\xi_{j}\right), \Pi$ is the polytope of couplings $\left\{\pi \in \mathcal{M}_{N_{\xi}}\left(\mathbb{R}_{+}\right): \pi \mathbb{1}=\mu_{1}\right.$ and $\left.\pi^{T} \mathbb{1}=\mu_{2}\right\}$ with $\mathbb{1}=(1, \ldots, 1)^{T}, C \in \mathcal{M}_{N_{\xi}}\left(\mathbb{R}_{+}\right)$is the cost matrix (i.e. $C_{i, j}=\left\|\xi_{i}-\xi_{j}\right\|^{2}$ ), $\langle.,\rangle_{F}$ is the Frobenius inner product and $H(\pi) \in \mathbb{R}$ is the entropy

$$
H(\pi)=-\sum_{i, j=1}^{N_{\xi}} \pi_{i, j}\left(\log \left(\pi_{i, j}\right)-1\right)
$$

According to the Sinkhorn's theorem, the transportation plan can be written as $\pi=\operatorname{diag}(\alpha) K \operatorname{diag}(\beta)$ where $K_{i, j}=\exp \left(-\frac{C_{i, j}}{\gamma}\right)$. Using the Kullback-Leibler divergence [26], the optimal transportation plan is solved by the Sinkhorn-Knopp algorithm where $\pi$ is iteratively projected onto the affine constraint sets $\Pi_{1}=\{\pi \in$ $\left.\mathcal{M}_{N_{\xi}}\left(\mathbb{R}_{+}\right): \pi \mathbb{1}=\mu_{1}\right\}$ and $\Pi_{2}=\left\{\pi \in \mathcal{M}_{N_{\xi}}\left(\mathbb{R}_{+}\right): \pi^{T} \mathbb{1}=\mu_{2}\right\}$. 


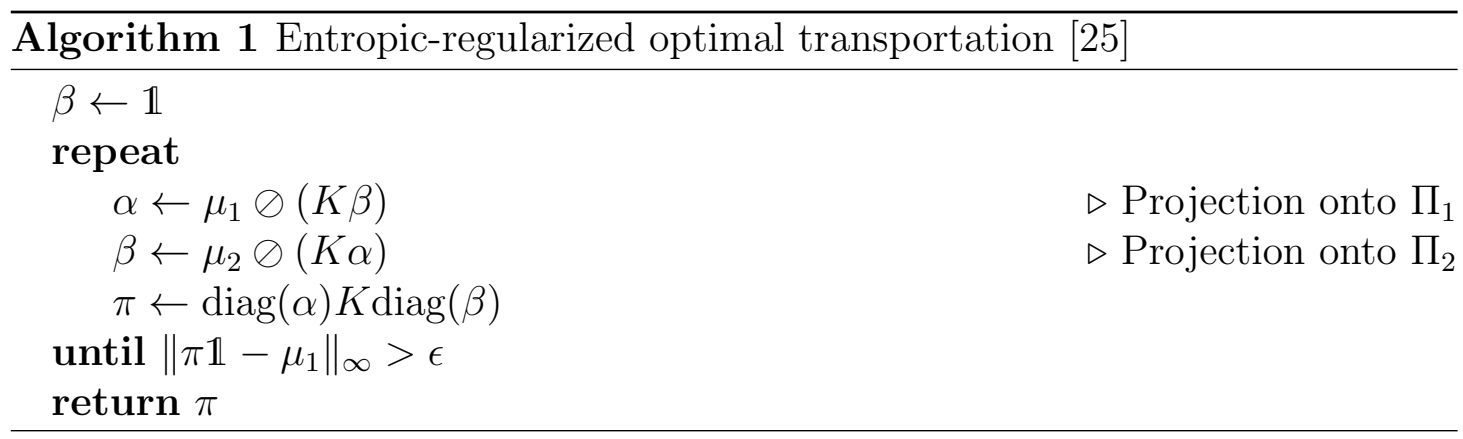

An application of the regularized optimal transport is the computation of Wasserstein barycenter. Given $N_{\text {simul }}$ high-fidelity snapshots $s_{l}$, the low-fidelity snapshot $s^{*}$ is the Wasserstein barycenter of the high-fidelity snapshots $s_{l}$

$$
\min _{s^{*} \in \mathbb{R}_{+}^{N_{\xi}}} \sum_{l=1}^{N_{s i m u l}} \lambda_{l} \mathcal{W}_{\gamma}\left(s_{l}, s^{*}\right)
$$

where $\sum_{l=1}^{N_{\text {simul }}} \lambda_{l}=1$. Before computing the low-fidelity snapshot $s^{*}$, we need to use 3D indexing to transform the velocity grid in a vector of size $N_{\xi}$ and we have to normalize the high-fidelity snapshots $s_{l}$ because they can have different densities $\rho_{l}$. After computing the barycenter, the high-fidelity snapshots $s_{l}$ recover their old densities $\rho_{l}$ and the low-fidelity snapshot $s^{*}$ is rescaled by $\sum_{l=1}^{N_{\text {simul }}} \lambda_{l} \rho_{l}$.

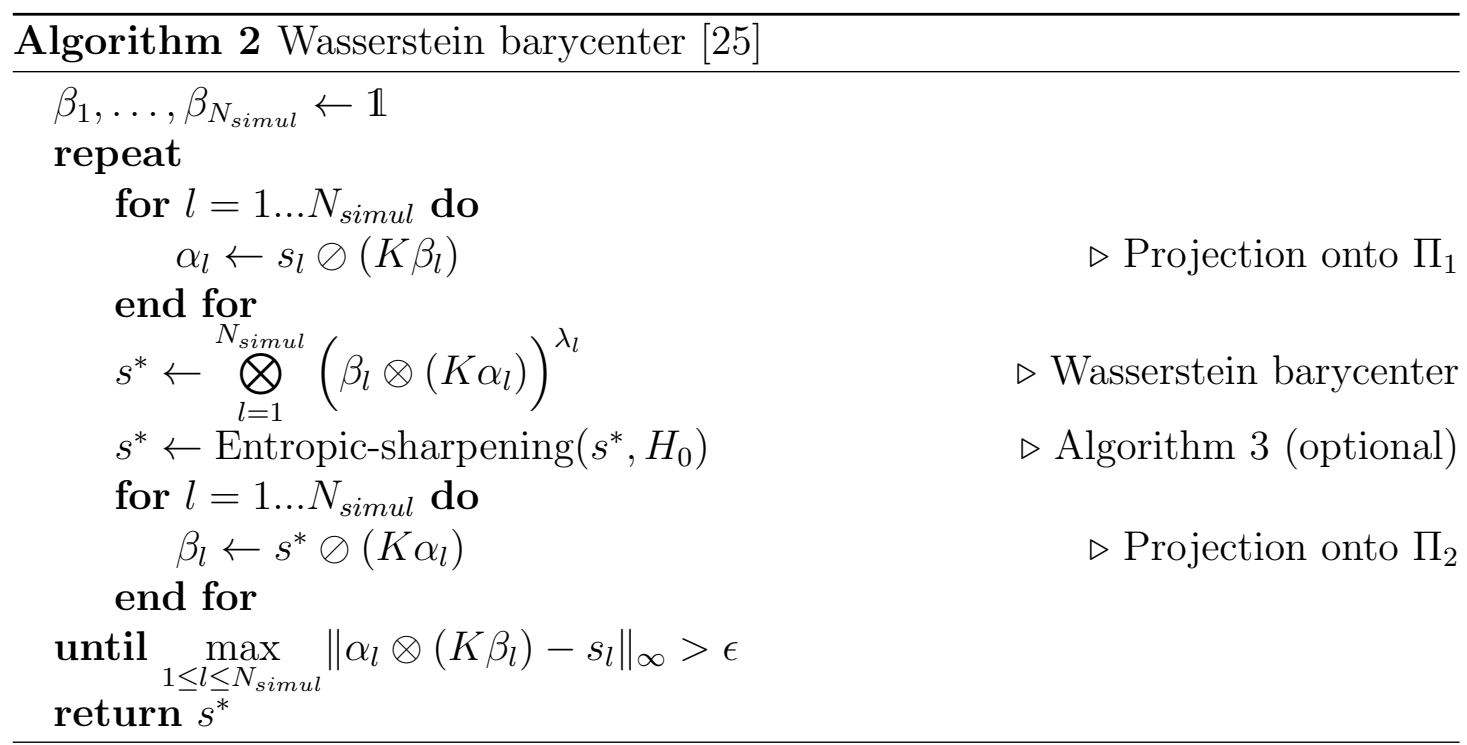

The main drawback of the entropic regularization of optimal transport method is that the barycenter appears too diffuse. To cure this issue, a constraint $H_{0} \in \mathbb{R}$ 
on the entropy is added as explained in [25]

$$
\left\{\begin{array}{l}
\min _{\substack{s^{*} \in \mathbb{R}_{+}^{N_{\xi}} \\
\text { s.t. }}} \sum_{l=1}^{N_{\text {simul }}} \lambda_{l} \mathcal{W}_{\gamma}\left(s_{l}, s^{*}\right) \\
H(q) \leq H_{0}
\end{array}\right.
$$

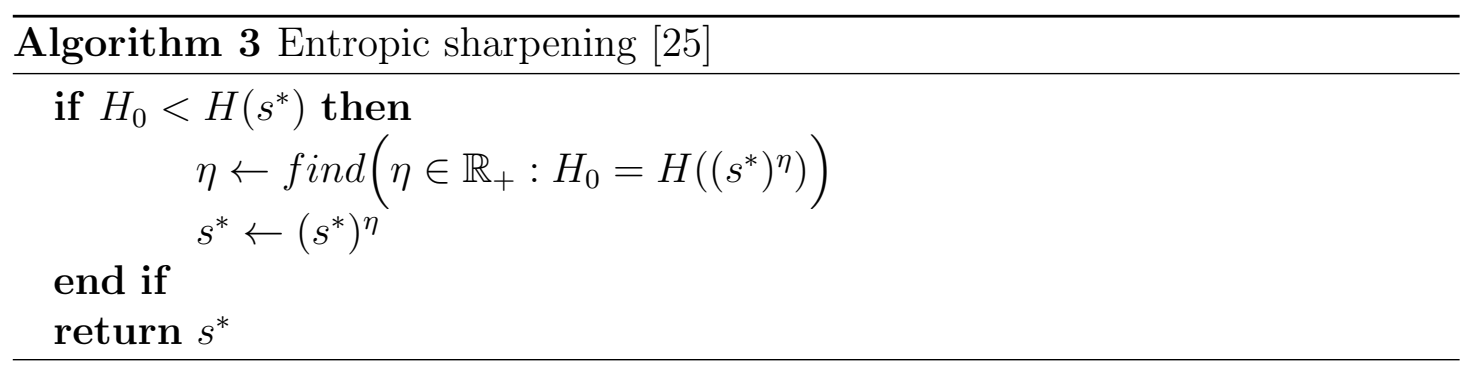

The function find is given in [27] and we choose $H_{0}=\max _{1 \leq l \leq N_{\text {simul }}} H\left(s_{l}\right), \gamma=5 \times 10^{-4}$ and $\epsilon=10^{-4}$ from the experiments. Figure 2 shows an example in 1D of low-fidelity snapshots $s^{*}$ obtained from a set of two high-fidelity snapshots $s_{1}$ and $s_{2}$.

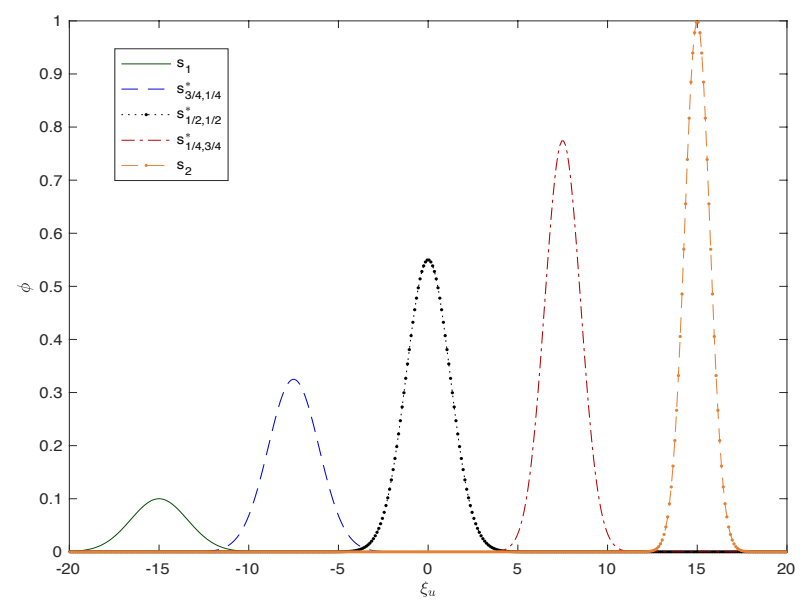

Figure 2: Wasserstein barycenters $s_{\lambda_{1}, \lambda_{2}}^{*}$ at barycentric coordinates $\left\{\left(s_{1}, \lambda_{1}\right),\left(s_{2}, \lambda_{2}\right)\right\}$.

To speed up the computations, the matrix-vector multiplications $K \alpha_{l}$ and $K \beta_{l}$ are replaced by a convolution with the gaussian kernel $K$ because the velocity space is discretized by an uniform cartesian grid. Moreover, this kernel is separable and the convolution are written as successive 1D convolutions leading to faster computations. The computational time of the optimal transport algorithm is evaluated with respect to the cost of the high-fidelity model for one time step and one space point. In this respect, we included the cost of interpolating a snapshot of the density distribution function $f$ and a snapshot of the discrete Maxwellian distribution function $\bar{M}_{f}$. Over 100 different runs, the computational time of Algorithm 2 is in average approximately the half of the one of the high-fidelity model. 
The overall run time of the low-fidelity snapshot procedure will also depend on the strategy adopted to enrich the database: only snapshots which will add new information to the snapshot database are created. In addition, the optimal transportation run time can be greatly improved by using GPU acceleration [28, 29]. Moreover, all low-fidelity snapshots can be independently computed in parallel while the high-fidelity snapshots must be computed sequentially in time.

\subsubsection{Proper Orthogonal Decomposition}

The basis functions $\Phi_{n}$ are built by POD to be the best representation in the least squares sense of the high-fidelity and low-fidelity snapshots contained in the database $S$. For simplicity, the POD is presented in the case (11) where the database contains only snapshots of the density distribution function $f$.

\subsubsection{Optimality of the basis functions.}

Let $f\left(\mathbf{x}_{i(l), j(l), k(l)}, \xi, t_{p(l)}\right)$ be a snapshot of the density distribution function $f$ taken at point $\mathbf{x}_{i(l), j(l), k(l)}$ and time $t_{p(l)}$. Given a database of $N_{\text {snaps }}$ snapshots, the subspace spanned by the basis functions $\Phi_{n}$ is the subspace of rank $N_{\text {pod }}$ minimizing in the least squares sense the difference between the snapshots and their projections $\mathcal{P}_{f}$ onto this subspace

$$
\left\{\begin{array}{c}
\underset{\Phi_{1}(\xi) \ldots \Phi_{N_{p o d}}(\xi)}{\operatorname{minimize}} \sum_{l=1}^{N_{\text {snaps }}}\left\|f\left(\mathbf{x}_{i(l), j(l), k(l)}, \xi, t_{p(l)}\right)-\mathcal{P}_{f}\left(\mathbf{x}_{i(l), j(l), k(l)}, \xi, t_{p(l)}\right)\right\|_{\xi}^{2} \\
\text { subject to } \\
\forall n, m \in \llbracket 1, N_{\text {pod }} \rrbracket,\left\langle\Phi_{n}, \Phi_{m}\right\rangle_{\xi}=\delta_{n, m}
\end{array}\right.
$$

where $\mathcal{P}_{f}(\mathbf{x}, \xi, t)=\sum_{n=1}^{N_{\text {pod }}}\left\langle f\left(\mathbf{x}, \xi^{\prime}, t\right), \Phi_{n}\left(\xi^{\prime}\right)\right\rangle_{\xi^{\prime}} \Phi_{n}(\xi)$. The snapshots $f_{l}(\xi)=$ $f\left(\mathbf{x}_{i(l), j(l), k(l)}, \xi, t_{p(l)}\right)$ are stored in matrix $S \in \mathcal{M}_{N_{\xi} \times N_{\text {snaps }}}(\mathbb{R})$

$$
S=\left(\begin{array}{cccc}
f_{1}\left(\xi_{1}\right) & f_{2}\left(\xi_{1}\right) & \cdots & f_{N_{\text {snaps }}}\left(\xi_{1}\right) \\
f_{1}\left(\xi_{2}\right) & f_{2}\left(\xi_{2}\right) & \cdots & f_{N_{\text {snaps }}}\left(\xi_{2}\right) \\
\vdots & \vdots & \ddots & \vdots \\
f_{1}\left(\xi_{N_{\xi}}\right) & f_{2}\left(\xi_{N_{\xi}}\right) & \cdots & f_{N_{\text {snaps }}}\left(\xi_{N_{\xi}}\right)
\end{array}\right)
$$

where we use 3D indexing to transform the velocity grid to a vector of size $N_{\xi}$. If the database contains low-fidelity snapshots or high-fidelity snapshots of $\bar{M}_{f}$, these snapshots are added as new columns to $S$. The basis functions $\Phi_{n}$ are stored in the matrix $\Phi \in \mathcal{M}_{N_{\xi} \times N_{\text {pod }}}(\mathbb{R})$

$$
\Phi=\left(\begin{array}{cccc}
\Phi_{1}\left(\xi_{1}\right) & \Phi_{2}\left(\xi_{1}\right) & \cdots & \Phi_{N_{p o d}}\left(\xi_{1}\right) \\
\Phi_{1}\left(\xi_{2}\right) & \Phi_{2}\left(\xi_{2}\right) & \cdots & \Phi_{N_{p o d}}\left(\xi_{2}\right) \\
\vdots & \vdots & \ddots & \vdots \\
\Phi_{1}\left(\xi_{N_{\xi}}\right) & \Phi_{2}\left(\xi_{N_{\xi}}\right) & \cdots & \Phi_{N_{p o d}}\left(\xi_{N_{\xi}}\right)
\end{array}\right)
$$


The discrete inner product is represented by the matrix $W \in \mathcal{M}_{N_{\xi}}(\mathbb{R})$ (i.e. $\left.\left\langle\Phi_{n}(\xi), \Phi_{m}(\xi)\right\rangle_{\xi}=\Phi_{n}^{T}(\xi) W \Phi_{m}(\xi)\right)$. The integrals are approximated by the rectangle rule and the associated matrix $W$ is given in section 4.1.1 by

$$
W=\operatorname{diag}(\Delta \xi, \ldots, \Delta \xi)=\left(\begin{array}{ccc}
\Delta \xi & & 0 \\
& \ddots & \\
0 & & \Delta \xi
\end{array}\right)
$$

Using the previous notations, the problem (16) can be written

$$
\left\{\begin{array}{cc}
\min _{\Phi} & \left\|S-\Phi \Phi^{T} W S\right\|_{F_{\xi}}^{2} \\
\text { s.t. } & \Phi^{T} W \Phi=I d
\end{array}\right.
$$

where $\|A\|_{F_{\xi}}^{2}=\operatorname{tr}\left(A^{T} W A\right)$. The solution of this problem is given by the EckartYoung theorem 1 .

Theorem 1. Let $S \in \mathcal{M}_{n \times m}(\mathbb{R})$ be a real rectangular matrix with $n \leq m$. Suppose that the singular value decomposition of $S$ is

$$
S=U \Sigma V^{T}
$$

where $U \in \mathcal{M}_{n}(\mathbb{R})$ and $V^{T} \in \mathcal{M}_{m}(\mathbb{R})$ are orthogonal matrices, and $\Sigma \in \mathcal{M}_{n \times m}(\mathbb{R})$ is a diagonal matrix with the singular values sorted in descending order. The best rank $k$ approximation to $S$ is given by

$$
\min _{\operatorname{rank}(X) \leq k}\|S-X\|_{F}^{2}=\left\|S-S^{*}\right\|_{F}^{2}=\sum_{i=k+1}^{n} \sigma_{i}^{2}
$$

where $\|\cdot\|_{F}$ is the Frobenius norm and $S^{*}$ is the trounced singular values decomposition of $S$

$$
S^{*}=\left(\begin{array}{ccc}
U_{1,1} & \cdots & U_{1, k} \\
\vdots & & \vdots \\
U_{n, 1} & \cdots & U_{n, k}
\end{array}\right)\left(\begin{array}{ccc}
\sigma_{1} & & 0 \\
& \ddots & \\
0 & & \sigma_{k}
\end{array}\right)\left(\begin{array}{ccc}
V_{1,1}^{T} & \cdots & V_{1, m}^{T} \\
\vdots & & \vdots \\
V_{k, 1}^{T} & \cdots & V_{k, m}^{T}
\end{array}\right) \in \mathcal{M}_{n, m}(\mathbb{R})
$$

By defining $\widehat{S}=\left(W^{\frac{1}{2}}\right)^{T} S$, the basis functions $\Phi_{n}$ are given by

$$
\Phi=\left(W^{\frac{1}{2}}\right)^{-T}\left(\begin{array}{ccc}
U_{1,1} & \cdots & U_{1, N_{p o d}} \\
\vdots & & \vdots \\
U_{N_{\xi}, 1} & \cdots & U_{N_{\xi}, N_{p o d}}
\end{array}\right)
$$

where $\widehat{S}=U \Sigma V^{T}$ is the SVD of $\widehat{S}$ and $W=W^{\frac{1}{2}}\left(W^{\frac{1}{2}}\right)^{T}$ is the Cholesky decomposition of $W$. 


\subsubsection{Choice of the number of basis functions.}

In the minimisation problem (16), the objective function is equivalent to

$$
\underset{\Phi_{1}(\xi) \ldots \Phi_{N_{\text {pod }}}(\xi)}{\operatorname{maximize}} \sum_{l=1}^{N_{\text {snaps }}} \sum_{n=1}^{N_{\text {pod }}}\left\langle f\left(\mathbf{x}_{i(l), j(l), k(l)}, \xi, t_{p(l)}\right), \Phi_{n}(\xi)\right\rangle_{\xi}^{2}
$$

By writing this problem in matrix form, the Eckart-Young theorem 1 gives

$$
\max _{\Phi}\left\|S^{T} W \Phi\right\|_{F}^{2}=\sum_{n=1}^{N_{\text {pod }}} \sigma_{n}^{2}
$$

where $\sigma_{n}$ are the singular values of $\widehat{S}$ sorted in descending order. The basis functions $\Phi_{n}$ are also optimal to capture the most energy $E_{\text {pod }}$ as possible

$$
E_{\text {pod }}=\sum_{n=1}^{N_{p o d}} \sigma_{n}^{2}
$$

Given a threshold $\epsilon$, the number of basis functions $N_{\text {pod }}$ is chosen to have at least $\epsilon$ of total energy captured by the basis

$$
\frac{\sum_{n=1}^{N_{p o d}} \sigma_{n}^{2}}{\sum_{n=1}^{N_{\xi}} \sigma_{n}^{2}}>\epsilon
$$

In practice, $\sigma_{n}$ decrease quickly and a small number of basis functions $N_{\text {pod }}$ is sufficient to exceed high threshold (i.e. $\epsilon \approx 99.9 \%$ ). We thus have a significant reduction of the number of degrees of freedom because $N_{\text {pod }} \ll N_{\xi}$.

\subsubsection{Construction of the basis functions.}

To build the basis functions $\Phi_{n}$, the number of snapshots can be in practice too large $\left(N_{\text {snaps }} \approx 10^{7}\right)$ to find the singular value decomposition of $\widehat{S}$. By considering the correlation matrix $\widehat{S} \widehat{S}^{T} \in \mathcal{M}_{N_{\xi}}(\mathbb{R})$, the left and right eigenvectors of this matrix are

$$
\widehat{S} \widehat{S}^{T}=\left(U \Sigma V^{T}\right)\left(U \Sigma V^{T}\right)^{T}=U \Sigma V^{T} V \Sigma U^{T}=U \Sigma^{2} U^{T}
$$

$U$ can be obtained by finding the eigendecomposition or the SVD of $\widehat{S} \widehat{S}^{T}$. We use the SVD of $\widehat{S} \widehat{S}^{T}$ to compute $U$ because this method is more accurate for small singular values. Figure 3 shows examples of basis functions $\Phi_{n}$ obtained from a snapshot database $S$ with this method. 

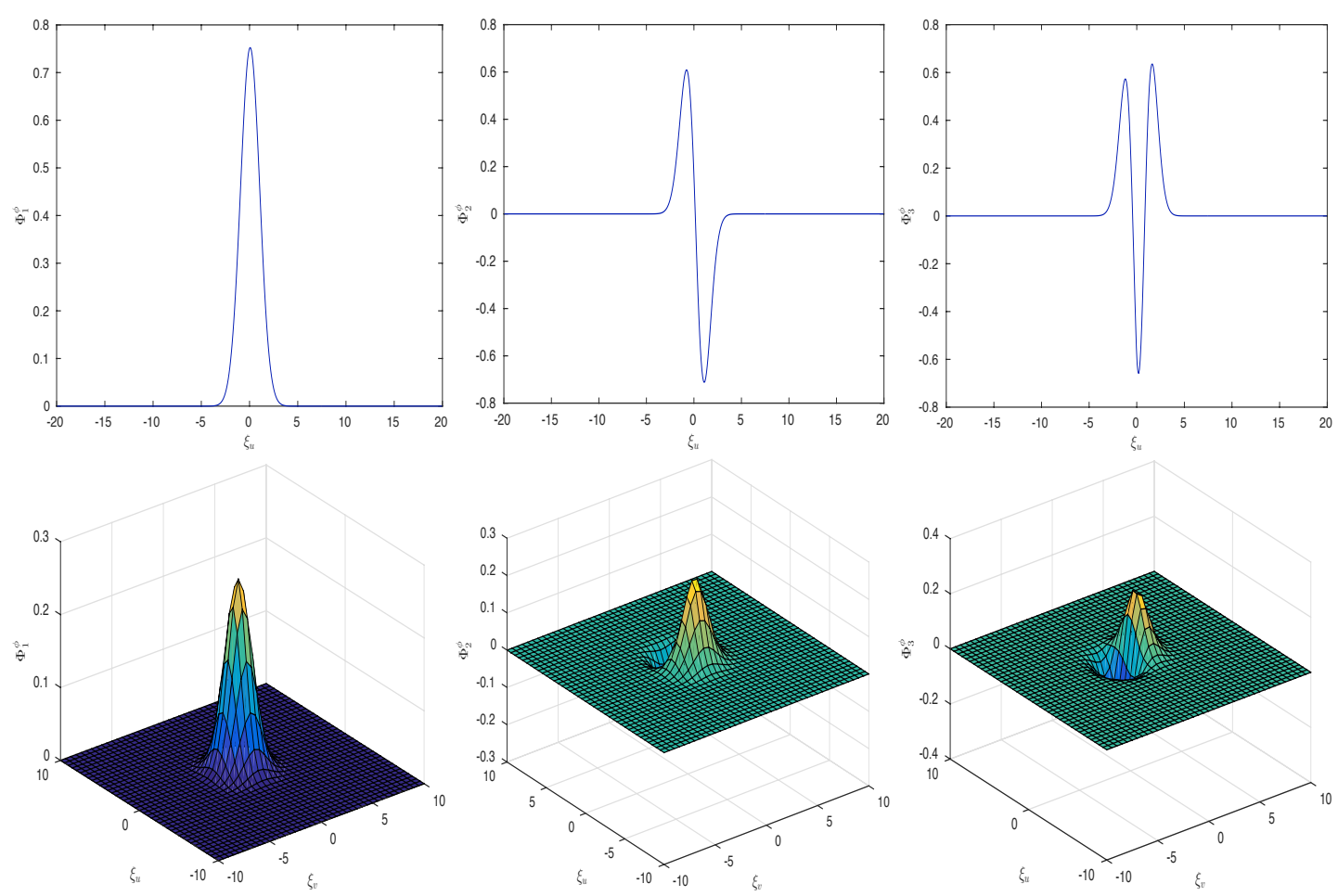

Figure 3: Examples of basis functions for $\phi$ in 1D (top) and in 2D (bottom).

The first basis function $\Phi_{1}^{\phi}$ (on the left) is close to the mean of the snapshots. In this case, the snapshots (see Figure 1) are close to Maxwellian distribution functions and $\Phi_{1}^{\phi}$ have the form of a Maxwellian distribution function. The other basis functions are small perturbations to have better approximation of the snapshots.

\subsection{Approximate density distribution function}

Once the basis functions $\Phi_{n}$ are built, the approximate density distribution function $\widetilde{f}$ depends only on the coefficients $a_{n}$. These coefficients $a_{n}$ are computed online by the Galerkin method.

\subsubsection{Galerkin method}

In the Galerkin method, the approximate density distribution function $\tilde{f}$ is inserted into the equation of the high-fidelity model (6) and leads to the residual

$$
\mathcal{R}(\mathbf{x}, \xi, t)=\frac{\partial \widetilde{f}}{\partial t}(\mathbf{x}, \xi, t)+\xi \cdot \nabla_{\mathbf{x}} \widetilde{f}(\mathbf{x}, \xi, t)-\frac{\bar{M}_{f}(\mathbf{x}, \xi, t)-\widetilde{f}(\mathbf{x}, \xi, t)}{\tau(\mathbf{x}, t)}
$$

This residual $\mathcal{R}$ is taken orthogonal to the basis functions $\Phi_{n}$. By projecting the residual onto the basis functions $\Phi_{n}$, we obtain the system $\forall n \in \llbracket 1, N_{\text {pod }} \rrbracket$

$$
\frac{\partial a_{n}}{\partial t}(\mathbf{x}, t)+\sum_{m=1}^{N_{p o d}}\left(A_{n, m} \frac{\partial a_{m}}{\partial x}(\mathbf{x}, t)+\AA_{n, m} \frac{\partial a_{m}}{\partial y}(\mathbf{x}, t)+\stackrel{A}{A}, m \frac{\partial a_{m}}{\partial z}(\mathbf{x}, t)\right)=\frac{a_{n}^{M}(\mathbf{x}, t)-a_{n}(\mathbf{x}, t)}{\tau(\mathbf{x}, t)}
$$


where $A_{n, m}=\int_{\mathbb{R}^{3}} \xi_{u} \Phi_{n}(\xi) \Phi_{m}(\xi) \mathrm{d} \xi, \stackrel{\circ}{A}_{n, m}=\int_{\mathbb{R}^{3}} \xi_{v} \Phi_{n}(\xi) \Phi_{m}(\xi) \mathrm{d} \xi, \stackrel{\star}{A}_{n, m}=\int_{\mathbb{R}^{3}} \xi_{w} \Phi_{n}(\xi) \Phi_{m}(\xi) \mathrm{d} \xi$ and $a_{n}^{M}(\mathbf{x}, t)=\int_{\mathbb{R}^{3}} \bar{M}_{f}(\mathbf{x}, \xi, t) \Phi_{n}(\xi) \mathrm{d} \xi$. In matrix form, this system reads

$$
\frac{\partial a}{\partial t}(\mathbf{x}, t)+A \frac{\partial a}{\partial x}(\mathbf{x}, t)+\AA \frac{\partial a}{\partial y}(\mathbf{x}, t)+\stackrel{\star}{A} \frac{\partial a}{\partial z}(\mathbf{x}, t)=\frac{a^{M}(\mathbf{x}, t)-a(\mathbf{x}, t)}{\tau(\mathbf{x}, t)}
$$

where $a=\left(a_{1}, \ldots, a_{N_{p o d}}\right)^{T}$ and $a^{M}=\left(a_{1}^{M}, \ldots, a_{N_{p o d}}^{M}\right)^{T}$.

Definition 1. The system (18) is hyperbolic if $A, \stackrel{\circ}{A}$ and $\stackrel{\star}{A}$ are diagonalizable with real eigenvalues.

The matrices $A, \AA$ and $\stackrel{\star}{A}$ are symmetric and thus diagonalizable by a real orthogonal similarity. According to Definition 1, this system is hyperbolic and the equations can be decoupled direction by direction with a linear change of variables. Let the eigendecompositions be $A=P D P^{T}$ where $D \in \mathcal{M}_{N_{\text {pod }}}(\mathbb{R})$ is a diagonal matrix and $P \in \mathcal{M}_{N_{\text {pod }}}(\mathbb{R})$ is an orthogonal matrix, the system 18 becomes

$$
\frac{\partial a}{\partial t}(\mathbf{x}, t)+P D \frac{\partial b}{\partial x}(\mathbf{x}, t)+\stackrel{\circ}{P} \stackrel{\circ}{D} \frac{\partial c}{\partial y}(\mathbf{x}, t)+\stackrel{\star}{P} \stackrel{\star}{D} \frac{\partial d}{\partial z}(\mathbf{x}, t)=\frac{a^{M}(\mathbf{x}, t)-a(\mathbf{x}, t)}{\tau(\mathbf{x}, t)}
$$

where the changes of variable are $b=P^{T} a, c=\stackrel{\circ}{P}^{T} a$ and $d=\stackrel{\star}{P}^{T} a$. In this form, the system (19) can be solved by a finite volume scheme in section 4.1.2. By considering the change of variables $b=P^{T} a$, the system $(19)$ is

$$
\frac{\partial b}{\partial t}(\mathbf{x}, t)+D \frac{\partial b}{\partial x}(\mathbf{x}, t)+P^{T} \stackrel{\AA}{P} \frac{\partial b}{\partial y}(\mathbf{x}, t)+P^{T} \stackrel{\star}{A} P \frac{\partial b}{\partial z}(\mathbf{x}, t)=\frac{b^{M}(\mathbf{x}, t)-b(\mathbf{x}, t)}{\tau(\mathbf{x}, t)}
$$

and the equations are decoupled for $D \frac{\partial b}{\partial x}$. In the same way by considering the system $(19)$ for $c$ (resp. $d)$, the equations will be decoupled for $\stackrel{\circ}{D} \frac{\partial c}{\partial y}\left(\operatorname{resp} . \stackrel{\star}{D} \frac{\partial d}{\partial z}\right)$.

\subsubsection{Approximate Maxwellian distribution function}

In the system of partial differential equations (19), the discrete Maxwellian distribution function $\bar{M}_{f}$ is projected onto the basis functions $\Phi_{n}$

$$
a_{n}^{M}(\mathbf{x}, t)=\left\langle\bar{M}(\mathbf{x}, \xi, t), \Phi_{n}(\xi)\right\rangle_{\xi}
$$

and the approximate Maxwellian distribution function $\widetilde{M}_{f}$ is given by

$$
\widetilde{M}_{f}(\mathbf{x}, \xi, t)=\sum_{n=1}^{N_{p o d}} a_{n}^{M}(\mathbf{x}, t) \Phi_{n}(\xi)
$$


Due to projection error, the approximate Maxwellian distribution function $\widetilde{M}_{f}$ does not necessarily verify equation (5)

$$
\left\langle\widetilde{M}_{f}(\mathbf{x}, \xi, t),\left(\begin{array}{c}
1 \\
\xi \\
\frac{\|\xi\|^{2}}{2}
\end{array}\right)\right\rangle_{\xi} \neq\left(\begin{array}{c}
\rho(\mathbf{x}, t) \\
\rho(\mathbf{x}, t) U(\mathbf{x}, t) \\
E(\mathbf{x}, t)
\end{array}\right)
$$

For this reason, the coefficients $a_{n}^{M}$ are not computed thanks to formula 20]. The approximate Maxwellian distribution function $\widetilde{M}_{f}$ is computed to conserve the mass, the momentum and the energy of the gas and to be as close as possible to the continuum Maxwellian $M_{f}$

$$
\left\{\begin{array}{l}
\underset{a^{M}(\mathbf{x}, t)}{\operatorname{minimize}}\left\|\widetilde{M}_{f}(\mathbf{x}, \xi, t)-M_{f}(\mathbf{x}, \xi, t)\right\|_{\xi}^{2} \\
\text { subject to }\left\langle\widetilde{M}_{f}(\mathbf{x}, \xi, t),\left(\begin{array}{c}
1 \\
\xi \\
\frac{\|\xi\|^{2}}{2}
\end{array}\right)\right\rangle_{\xi}=\left(\begin{array}{c}
\rho(\mathbf{x}, t) \\
\rho(\mathbf{x}, t) U(\mathbf{x}, t) \\
E(\mathbf{x}, t)
\end{array}\right)
\end{array}\right.
$$

The approximate Maxwellian distribution function $\widetilde{M}$ verifying this minimisation problem is given by the theorem 2 .

Theorem 2. Let $A \in \mathcal{M}_{m \times n}(\mathbb{R})$ be a rectangular matrix such that $A^{T} A=I d$, $C \in \mathcal{M}_{k \times n}(\mathbb{R})$ be a rectangular matrix, $b \in \mathbb{R}^{m}$ and $d \in \mathbb{R}^{k}$. The solution of

$$
\left\{\begin{array}{lc}
\min _{X \in \mathbb{R}^{n}} & \frac{1}{2}\|A X-b\|^{2} \\
\text { s.t. } & C X=d
\end{array}\right.
$$

is

$$
X^{*}=A^{T} b+C^{+}\left(d-C A^{T} b\right)
$$

where $C^{+}$is the Moore-Penrose inverse of $C$.

Proof. The solution of this minimisation problem is given by the method of Lagrange multipliers

$$
X^{*}=\left(A^{T} A\right)^{-1} A^{T} b+\left(A^{T} A\right)^{-1} C^{T}\left(C\left(A^{T} A\right)^{-1} C^{T}\right)^{-1}\left(d-C\left(A^{T} A\right)^{-1} A^{T} b\right)
$$

By using $A^{T} A=I d$, the solution becomes

$$
X^{*}=A^{T} b+C^{T}\left(C C^{T}\right)^{-1}\left(d-C A^{T} b\right)
$$

If $C C^{T}$ is non-invertible, there is no solution satisfying the constraints. In this case, we replace the equality constraints $C X=d$ by $C X=C C^{+} d$, which is equivalent to search the best approximation in the least squares sense of the constraints $C X=d$ minimizing $\frac{1}{2}\|A X-b\|^{2}$. The corresponding solution is given by

$$
X^{*}=A^{T} b+C^{+}\left(d-C A^{T} b\right)
$$

When $C C^{T}$ is invertible, this expression is still valid because $C^{+}=C^{T}\left(C C^{T}\right)^{-1}$. 
In the problem 22 , the objective function is a $N_{\xi} \times N_{\text {pod }}$ system usually overdetermined

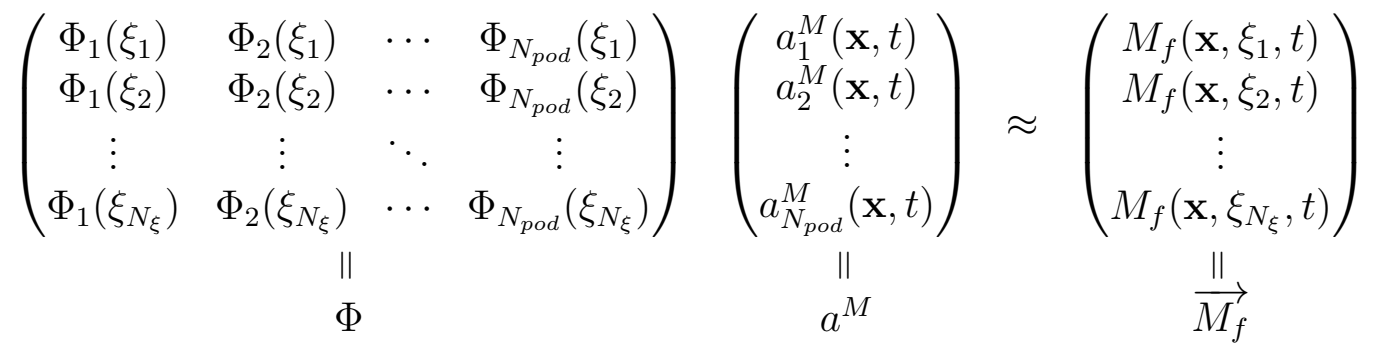

and the equality constraints are a $5 \times N_{\text {pod }}$ system generally underdetermined

$$
\left(\begin{array}{cccc}
\left\langle\Phi_{1}(\xi), 1\right\rangle_{\xi} & \left\langle\Phi_{2}(\xi), 1\right\rangle_{\xi} & \cdots & \left\langle\Phi_{N_{p o d}}(\xi), 1\right\rangle_{\xi} \\
\left\langle\Phi_{1}(\xi), \xi_{u}\right\rangle_{\xi} & \left\langle\Phi_{2}(\xi), \xi_{u}\right\rangle_{\xi} & \cdots & \left\langle\Phi_{N_{p o d}}(\xi), \xi_{u}\right\rangle_{\xi} \\
\left\langle\Phi_{1}(\xi), \xi_{v}\right\rangle_{\xi} & \left\langle\Phi_{2}(\xi), \xi_{v}\right\rangle_{\xi} & \cdots & \left\langle\Phi_{N_{p o d}}(\xi), \xi_{v}\right\rangle_{\xi} \\
\left\langle\Phi_{1}(\xi), \xi_{w}\right\rangle_{\xi} & \left\langle\Phi_{2}(\xi), \xi_{w}\right\rangle_{\xi} & \cdots & \left\langle\Phi_{N_{p o d}}(\xi), \xi_{w}\right\rangle_{\xi} \\
\left\langle\Phi_{1}(\xi), \frac{\|\xi\|^{2}}{2}\right\rangle_{\xi} & \left\langle\Phi_{2}(\xi), \frac{\|\xi\|^{2}}{2}\right\rangle_{\xi} & \cdots & \left\langle\Phi_{N_{p o d}}(\xi), \frac{\|\xi\|^{2}}{2}\right\rangle_{\xi}
\end{array}\right)\left(\begin{array}{c}
a_{1}^{M}(\mathbf{x}, t) \\
a_{2}^{M}(\mathbf{x}, t) \\
\vdots \\
\| \\
\Psi
\end{array}\right.
$$

Using the previous notations and the theorem 2, the coefficients $a^{M}$ are computed at any point in space and every time step at cost $O\left(N_{\xi} N_{\text {pod }}\right)$ by the formula

$$
a^{M}(\mathbf{x}, t)=\Phi^{T} W \overrightarrow{M_{f}}(\mathbf{x}, t)+\Psi^{+}\left(\vec{\rho}(\mathbf{x}, t)-\Psi \Phi^{T} W \overrightarrow{M_{f}}(\mathbf{x}, t)\right)
$$

where $\Psi^{+}$is the Moore-Penrose inverse of $\Psi$. The matrices $\Phi^{T} W, \Psi^{+}$and $\Psi \Phi^{T} W$ do not depend on $\mathbf{x}$ and $t$, and they can be pre-calculated to save computing time. The approximate Maxwellian distribution function depends on the density $\rho$, the macroscopic velocity $U$ and the temperature $T$ of the gas and in the following, $a^{M}[\rho, U, T]$ are the coefficients of the approximate Maxwellian distribution function corresponding to $(\rho, U, T)$. In $1 \mathrm{D}$ and $2 \mathrm{D}$, we use the same idea by redefining $\Phi$, $\Psi, a^{M}, \overrightarrow{M_{f}}$ and $\vec{\rho}$. In $1 \mathrm{D}$, the objective function is the $\left(2 N_{\xi}\right) \times\left(N_{\text {pod }}^{\phi}+N_{\text {pod }}^{\psi}\right)$ system

$$
\left(\begin{array}{cccccc}
\Phi_{1}^{\phi}\left(\xi_{u_{1}}\right) & \cdots & \Phi_{N_{\text {pod }}^{\phi}}^{\phi}\left(\xi_{u_{1}}\right) & 0 & \cdots & 0 \\
\vdots & & \vdots & \vdots & & \vdots \\
\Phi_{1}^{\phi}\left(\xi_{u_{N_{\xi}}}\right) & \cdots & \Phi_{N_{\text {pod }}^{\phi}}^{\phi}\left(\xi_{u_{N_{\xi}}}\right) & 0 & \cdots & 0 \\
0 & \cdots & 0 & \Phi_{1}^{\psi}\left(\xi_{u_{1}}\right) & \cdots & \Phi_{N_{\text {pod }}^{\psi}}^{\psi}\left(\xi_{u_{1}}\right) \\
\vdots & & \vdots & \vdots & & \vdots \\
0 & \cdots & 0 & \Phi_{1}^{\psi}\left(\xi_{u_{N_{\xi}}}\right) & \cdots & \Phi_{N_{\text {pod }}^{\psi}}^{\psi}\left(\xi_{u_{N_{\xi}}}\right)
\end{array}\right)\left(\begin{array}{c}
a_{1}^{M_{\phi}}(x, t) \\
\vdots \\
a_{N_{\phi o d}^{\phi}}^{M_{\phi}(x, t)} \\
a_{1}^{M M_{\psi}}(x, t) \\
\vdots \\
a_{N_{p o d}^{\psi}}^{M_{\psi}^{\psi}}(x, t)
\end{array}\right) \approx\left(\begin{array}{c}
M_{\phi}\left(x, \xi_{u_{1}}, t\right) \\
\vdots \\
M_{\phi}\left(x, \xi_{u_{N_{\xi}}}, t\right) \\
M_{\psi}\left(x, \xi_{u_{1}}, t\right) \\
\vdots \\
M_{\psi}\left(x, \xi_{u_{N_{\xi}}}, t\right)
\end{array}\right)
$$


and the equality constraints are the $3 \times\left(N_{\text {pod }}^{\phi}+N_{\text {pod }}^{\psi}\right)$ system

$$
\left(\begin{array}{cccccc}
\left\langle\Phi_{1}^{\phi}, 1\right\rangle_{\xi} & \cdots & \left\langle\Phi_{N_{\text {pod }}^{\phi}}^{\phi}, 1\right\rangle_{\xi} & 0 & \cdots & 0 \\
\left\langle\Phi_{1}^{\phi}, \xi_{u}\right\rangle_{\xi} & \cdots & \left\langle\Phi_{N_{\text {pod }}^{\phi}}^{\phi}, \xi_{u}\right\rangle_{\xi} & 0 & \cdots & 0 \\
\left\langle\Phi_{1}^{\phi}, \frac{\xi_{u}^{2}}{2}\right\rangle_{\xi} & \cdots & \left\langle\Phi_{N_{\text {pod }}^{\phi}}^{\phi}, \frac{\xi_{u}^{2}}{2}\right\rangle_{\xi} & \left\langle\Phi_{1}^{\psi}, 1\right\rangle_{\xi} & \cdots & \left\langle\Phi_{N_{\text {pod }}^{\psi}}^{\psi}, 1\right\rangle_{\xi}
\end{array}\right)\left(\begin{array}{c}
a_{1}^{M_{\phi}}(x, t) \\
\vdots \\
a_{N_{\phi o d}}^{M_{\phi}}(x, t) \\
a_{1}^{M}(x, t) \\
\vdots \\
a_{N_{\text {pod }}^{\psi}}^{M_{\psi}}(x, t)
\end{array}\right)=\left(\begin{array}{c}
\rho(x, t) \\
\rho(x, t) u(x, t) \\
E(x, t)
\end{array}\right)
$$

In $2 \mathrm{D}$, the objective function is the $\left(2 N_{\xi}\right) \times\left(N_{\text {pod }}^{\phi}+N_{\text {pod }}^{\psi}\right)$ system

$$
\left(\begin{array}{cccccc}
\Phi_{1}^{\phi}\left(\xi_{2_{1}}\right) & \cdots & \Phi_{N_{\text {pod }}^{\phi}}^{\phi}\left(\xi_{2_{1}}\right) & 0 & \cdots & 0 \\
\vdots & & \vdots & \vdots & & \vdots \\
\Phi_{1}^{\phi}\left(\xi_{2_{N_{\xi}}}\right) & \cdots & \Phi_{N_{p o d}^{\phi}}^{\phi}\left(\xi_{2_{N_{\xi}}}\right) & 0 & \cdots & 0 \\
0 & \cdots & 0 & \Phi_{1}^{\psi}\left(\xi_{2_{1}}\right) & \cdots & \Phi_{N_{\text {pod }}^{\psi}}^{\psi}\left(\xi_{2_{1}}\right) \\
\vdots & & \vdots & \vdots & & \vdots \\
0 & \cdots & 0 & \Phi_{1}^{\psi}\left(\xi_{2_{N_{\xi}}}\right) & \cdots & \Phi_{N_{\text {pod }}^{\psi}}^{\psi}\left(\xi_{2_{N_{\xi}}}\right)
\end{array}\right)\left(\begin{array}{c}
a_{1}^{M_{\phi}}(\mathbf{x}, t) \\
\vdots \\
a_{N_{p o d}^{M_{\phi}}(\mathbf{x}, t)} \\
a_{1}^{M \psi}(\mathbf{x}, t) \\
\vdots \\
a_{N_{p o d}^{\psi}}^{M_{\psi}^{\psi}}(\mathbf{x}, t)
\end{array}\right) \approx\left(\begin{array}{c}
M_{\phi}\left(\mathbf{x}, \xi_{2_{1}}, t\right) \\
\vdots \\
M_{\phi}\left(\mathbf{x}, \xi_{2_{N_{\xi}}}, t\right) \\
M_{\psi}\left(\mathbf{x}, \xi_{2_{1}}, t\right) \\
\vdots \\
M_{\psi}\left(\mathbf{x}, \xi_{2_{N_{\xi}}}, t\right)
\end{array}\right)
$$

and the equality constraints are the $4 \times\left(N_{\text {pod }}^{\phi}+N_{\text {pod }}^{\psi}\right)$ system

$$
\left(\begin{array}{cccccc}
\left\langle\Phi_{1}^{\phi}, 1\right\rangle_{\xi} & \cdots & \left\langle\Phi_{N_{p o d}^{\phi}}^{\phi}, 1\right\rangle_{\xi} & 0 & \cdots & 0 \\
\left\langle\Phi_{1}^{\phi}, \xi_{u}\right\rangle_{\xi} & \cdots & \left\langle\Phi_{N_{\text {pod }}^{\phi}}^{\phi}, \xi_{u}\right\rangle_{\xi} & 0 & \cdots & 0 \\
\left\langle\Phi_{1}^{\phi}, \xi_{v}\right\rangle_{\xi} & \cdots & \left\langle\Phi_{N_{\text {pod }}^{\phi}}^{\phi}, \xi_{v}\right\rangle_{\xi} & 0 & \cdots & 0 \\
\left\langle\Phi_{1}^{\phi}, \frac{\left\|\xi_{2}\right\|^{2}}{2}\right\rangle_{\xi} & \cdots & \left\langle\Phi_{N_{\text {pod }}^{\phi}}^{\phi}, \frac{\left\|\xi_{2}\right\|^{2}}{2}\right\rangle_{\xi} & \left\langle\Phi_{1}^{\psi}, 1\right\rangle_{\xi} \cdots & \left\langle\Phi_{N_{\text {pod }}^{\psi}}^{\psi}, 1\right\rangle \\
\xi
\end{array}\right)\left(\begin{array}{c}
a_{1}^{M_{\phi}}(\mathbf{x}, t) \\
\vdots \\
a_{N_{p o d}}^{M_{\phi}}(\mathbf{x}, t) \\
a_{1}^{M}(\mathbf{x}, t) \\
\vdots \\
a_{N_{\text {pod }}^{\psi}}^{M_{\psi}}(\mathbf{x}, t)
\end{array}\right)=\left(\begin{array}{c}
\rho(\mathbf{x}, t) \\
\rho(\mathbf{x}, t) u(\mathbf{x}, t) \\
\rho(\mathbf{x}, t) v(\mathbf{x}, t) \\
E(\mathbf{x}, t)
\end{array}\right)
$$

With these redefinitions, the approximate Maxwellian distribution functions $\widetilde{M}_{\phi}$ and $\widetilde{M}_{\psi}$ are given by the formula $(23)$ in $1 \mathrm{D}$ and $2 \mathrm{D}$.

\section{Numerical methods for the reduced-order model}

This section describes the numerical schemes used to discretized the reducedorder model.

\subsection{Numerical scheme}

The numerical schemes for the physical space, the velocity space and the time are first-order schemes. 


\subsubsection{Velocity space discretization}

The velocity space $\Omega_{\xi}=\left[-L_{\xi_{u}}, L_{\xi_{u}}\right] \times\left[-L_{\xi_{v}}, L_{\xi_{v}}\right] \times\left[-L_{\xi_{w}}, L_{\xi_{w}}\right]$ is discretized by a cartesian grid. The lengths of the grid $L_{\xi_{u}}, L_{\xi_{v}}$ and $L_{\xi_{w}}$ are chosen to capture at least $99.99 \%$ of the integral of the density distribution function $f$. The velocity grid contains $N_{\xi}$ points $\xi_{i, j, k}=\left(\xi_{u_{i}}, \xi_{v_{j}}, \xi_{w_{k}}\right)$ where $\xi_{u_{i}}=-L_{\xi_{u}}+\left(i-\frac{1}{2}\right) \Delta \xi_{u}$ and $\Delta \xi_{u}=\frac{2 L_{\xi_{u}}}{N_{\xi_{u}}}$. The rectangle rules is used to approximate the integrals

$$
\int_{\Omega_{\xi}} g(\xi) \mathrm{d} \xi \approx \Delta \xi_{u} \Delta \xi_{v} \Delta \xi_{w} \sum_{i=1}^{N_{\xi_{u}}} \sum_{j=1}^{N_{\xi_{v}}} \sum_{k=1}^{N_{\xi_{w}}} g\left(\xi_{i, j, k}\right)
$$

By using 3D indexing (i.e. $\xi_{l}=\xi_{i(l), j(l), k(l)}$ ) and by defining $N_{\xi}=N_{\xi_{u}} N_{\xi_{v}} N_{\xi_{w}}$ and $\Delta \xi=\Delta \xi_{u} \Delta \xi_{v} \Delta \xi_{w}$, the discrete inner product is

$$
\left\langle g_{1}, g_{2}\right\rangle_{\xi}=\Delta \xi \sum_{l=1}^{N_{\xi}} g_{1}\left(\xi_{l}\right) g_{2}\left(\xi_{l}\right)
$$

and corresponds in matrix form to

$$
\left\langle g_{1}, g_{2}\right\rangle_{\xi}=\left(\begin{array}{llll}
g_{1}\left(\xi_{1}\right) & g_{1}\left(\xi_{2}\right) & \cdots & g_{1}\left(\xi_{N_{\xi}}\right)
\end{array}\right)\left(\begin{array}{ccc}
\Delta \xi & & 0 \\
& \ddots & \\
0 & & \Delta \xi
\end{array}\right)\left(\begin{array}{c}
g_{2}\left(\xi_{1}\right) \\
g_{2}\left(\xi_{2}\right) \\
\vdots \\
\\
g_{2}\left(\xi_{N_{\xi}}\right)
\end{array}\right)
$$

\subsubsection{Physical space discretization}

The physical space $\Omega_{\mathbf{x}}=\left[x_{\min }, x_{\max }\right] \times\left[y_{\min }, y_{\max }\right] \times\left[z_{\min }, z_{\max }\right]$ is discretized by a cartesian grid. The grid contains $N_{\mathbf{x}}$ points $\mathbf{x}_{i, j, k}=\left(x_{i}, y_{j}, z_{k}\right)$ where $x_{i}=$ $x_{\min }+\left(i-\frac{1}{2}\right) \Delta x$ and $\Delta x=\frac{x_{\max }-x_{\min }}{N_{x}}$. The grid contains also cells $\Omega_{\mathbf{x}_{i, j, k}}$ of center $\mathbf{x}_{i, j, k}$ and of size $\Delta x \times \Delta y \times \Delta z$. In the hyperbolic system (19), a finite volume scheme [19] is used for the convective term and a centered approximation is used for the collision term on each cell $\Omega_{\mathbf{x}_{i, j, k}}$

$\frac{\partial a_{\mathbf{x}_{i, j, k}}}{\partial t}(t)+P D \int_{\partial \Omega_{\mathbf{x}_{i, j, k}}} b(\mathbf{x}, t) n_{x} \mathrm{~d} \sigma+\stackrel{\circ}{P} \stackrel{\circ}{D} \int_{\partial \Omega_{\mathbf{x}_{i, j, k}}} c(\mathbf{x}, t) n_{y} \mathrm{~d} \sigma+\stackrel{ \pm}{P} \stackrel{ \pm}{D} \int_{\partial \Omega_{\mathbf{x}_{i, j, k}}} d(\mathbf{x}, t) n_{z} \mathrm{~d} \sigma=\frac{a_{\mathbf{x}_{i, j, k}}^{M}(t)-a_{\mathbf{x}_{i, j, k}}(t)}{\tau_{\mathbf{x}_{i, j, k}}(t)}$

where $a_{\mathbf{x}_{i, j, k}}(t)=\frac{1}{\left|\Omega_{\mathbf{x}_{i, j, k}}\right|} \int_{\Omega_{\mathbf{x}_{i, j, k}}} a(\mathbf{x}, t) \mathrm{d} \mathbf{x}$ and $n=\left(n_{x}, n_{y}, n_{z}\right)^{T} \in \mathbb{R}^{3}$ is the outward normal of the cell $\Omega_{\mathbf{x}_{i, j, k}}$. On cartesian grid, the finite volume scheme reads :

$$
D \int_{\partial \Omega_{\mathbf{x}_{i, j, k}}} b(\mathbf{x}, t) n_{x} \mathrm{~d} \sigma=\frac{F_{i+\frac{1}{2}, j, k}-F_{i-\frac{1}{2}, j, k}}{\Delta x}
$$


where the flux $F_{i+\frac{1}{2}, j, k}$ between cells $\Omega_{\mathbf{x}_{i, j, k}}$ and $\Omega_{\mathbf{x}_{i+1, j, k}}$ is

$$
F_{i+\frac{1}{2}, j, k}=\max (D, 0) b_{\mathbf{x}_{i, j, k}}(t)+\min (D, 0) b_{\mathbf{x}_{i+1, j, k}}(t)
$$

and $\max (D, 0)$ is the diagonal matrix $D$ with the negative elements replaced by 0 . Moreover $P$ and $D$ are constant and we can avoid the change of variable $b$

$$
\begin{aligned}
\int_{\Omega_{\mathbf{x}_{i, j}, k}} A \frac{\partial a}{\partial x}(\mathbf{x}, t) \mathrm{d} \mathbf{x} & =P D \int_{\partial \Omega_{\mathbf{x}_{i, j, k}}} b(\mathbf{x}, t) n_{x} \mathrm{~d} \sigma \\
& =P \frac{F_{i+\frac{1}{2}, j, k}-F_{i-\frac{1}{2}, j, k}}{\Delta x} \\
& =P\left(\max (D, 0) \frac{b_{\mathbf{x}_{i, j, k}}(t)-b_{\mathbf{x}_{i-1, j, k}}(t)}{\Delta x}+\min (D, 0) \frac{b_{\mathbf{x}_{i+1, j, k}}(t)-b_{\mathbf{x}_{i, j, k}}(t)}{\Delta x}\right) \\
& =P \max (D, 0) P^{T} \frac{a_{\mathbf{x}_{i, j, k}}(t)-a_{\mathbf{x}_{i-1, j, k}}(t)}{\Delta x}+P \min (D, 0) P^{T} \frac{a_{\mathbf{x}_{i+1, j, k}}(t)-a_{\mathbf{x}_{i, j, k}}(t)}{\Delta x}
\end{aligned}
$$

where the matrices $P \max (D, 0) P^{T}$ and $P \min (D, 0) P^{T}$ can be pre-calculated to save computing time. In the same way, the finite volume scheme for the variable $a$ is

$$
\begin{aligned}
& \int_{\Omega_{\mathbf{x}_{i, j, k}}} \frac{\partial a}{\partial y}(\mathbf{x}, t) \mathrm{d} \mathbf{x}=\stackrel{\circ}{P} \max (\stackrel{\circ}{D}, 0) \stackrel{\circ}{P}^{T} \frac{a_{\mathbf{x}_{i, j, k}}(t)-a_{\mathbf{x}_{i, j-1, k}}(t)}{\Delta y}+\stackrel{\circ}{P} \min (\stackrel{\circ}{D}, 0) \stackrel{\circ}{P}^{T} \frac{a_{\mathbf{x}_{i, j+1, k}}(t)-a_{\mathbf{x}_{i, j, k}}(t)}{\Delta y} \\
& \int_{\Omega_{\mathbf{x}_{i, j}, k}} \underset{\star}{A z} \frac{\partial a}{\partial}(\mathbf{x}, t) \mathrm{d} \mathbf{x}=\stackrel{\star}{P} \max (\stackrel{\star}{D}, 0) \stackrel{\star}{P} \frac{a_{\mathbf{x}_{i, j, k}}(t)-a_{\mathbf{x}_{i, j, k-1}}(t)}{\Delta z}+\stackrel{\star}{P} \min (\stackrel{\star}{D}, 0) \stackrel{\star}{P} T \frac{a_{\mathbf{x}_{i, j, k+1}}(t)-a_{\mathbf{x}_{i, j, k}}(t)}{\Delta z}
\end{aligned}
$$

The boundary conditions are given in the section 4.2 .

\subsubsection{Time discretization}

A first-order IMEX Runge-Kutta scheme [16, 17, 18] is used in time. In this scheme, the convective term is treated explicitly while the collision term is treated implicitly

$$
\frac{\partial a_{\mathbf{x}_{i, j, k}}}{\partial t}(t)+\underbrace{\int_{\Omega_{\mathbf{x}_{i, j, k}}} A \frac{\partial a}{\partial x}(\mathbf{x}, t)+\AA \frac{\partial a}{\partial y}(\mathbf{x}, t)+\AA \frac{\partial a}{\partial z}(\mathbf{x}, t) \mathrm{d} \mathbf{x}}_{\text {convective term }}=\underbrace{\frac{a_{\mathbf{x}_{i, j, k}}^{M}(t)-a_{\mathbf{x}_{i, j, k}}(t)}{\tau_{\mathbf{x}_{i, j, k}}(t)}}_{\text {collision term }}
$$

In this way, the time step $\Delta t$ doesn't depend on the collision term which can be very small due to $\tau_{\mathbf{x}_{i, j, k}}$. We have one intermediate step given by the implicit formula

$$
a_{\mathbf{x}_{i, j, k}}\left(t_{p+\frac{1}{2}}\right)=a_{\mathbf{x}_{i, j, k}}\left(t_{p}\right)+\Delta t \frac{a_{\mathbf{x}_{i, j, k}}^{M}\left(t_{p+\frac{1}{2}}\right)-a_{\mathbf{x}_{i, j, k}}\left(t_{p+\frac{1}{2}}\right)}{\tau_{\mathbf{x}_{i, j, k}}\left(t_{p+\frac{1}{2}}\right)}
$$

By integrating in velocity space this formula, $\widetilde{f}_{\mathbf{x}_{i, j, k}}\left(\xi, t_{p}\right)$ and $\widetilde{f}_{\mathbf{x}_{i, j, k}}\left(\xi, t_{p+\frac{1}{2}}\right)$ have the same moments because $\widetilde{M}_{\mathbf{x}_{i, j, k}}\left(\xi, t_{p+\frac{1}{2}}\right)$ and $\widetilde{f}_{\mathbf{x}_{i, j, k}}\left(\xi, t_{p+\frac{1}{2}}\right)$ have the same moments. Moreover $\widetilde{M}_{\mathbf{x}_{i, j, k}}\left(\xi, t_{p+\frac{1}{2}}\right)$ and $\widetilde{f}_{\mathbf{x}_{i, j, k}}\left(\xi, t_{p}\right)$ have the same moments, which 
implies that $\widetilde{M}_{\mathbf{x}_{i, j, k}}\left(\xi, t_{p+\frac{1}{2}}\right)$ is equal to $\widetilde{M}_{\mathbf{x}_{i, j, k}}\left(\xi, t_{p}\right)$. The explicit formula of the intermediate time step is

$$
a_{\mathbf{x}_{i, j, k}}\left(t_{p+\frac{1}{2}}\right)=\frac{\tau_{\mathbf{x}_{i, j, k}}\left(t_{p}\right)}{\Delta t+\tau_{\mathbf{x}_{i, j, k}}\left(t_{p}\right)}\left(a_{\mathbf{x}_{i, j, k}}\left(t_{p}\right)+\frac{\Delta t}{\tau_{\mathbf{x}_{i, j, k}}\left(t_{p}\right)} a_{\mathbf{x}_{i, j, k}}^{M}\left(t_{p}\right)\right)
$$

and the next time step is given by

$$
\begin{gathered}
a_{\mathbf{x}_{i, j, k}}\left(t_{p+1}\right)=a_{\mathbf{x}_{i, j, k}}\left(t_{p}\right)-\Delta t \int_{\Omega_{\mathbf{x}_{i, j, k}}} A \frac{\partial a}{\partial x}\left(\mathbf{x}, t_{p+\frac{1}{2}}\right)+\AA \frac{\partial a}{\partial y}\left(\mathbf{x}, t_{p+\frac{1}{2}}\right)+\stackrel{\star}{A} \frac{\partial a}{\partial z}\left(\mathbf{x}, t_{p+\frac{1}{2}}\right) \mathrm{d} \mathbf{x} \\
+\Delta t \frac{a_{\mathbf{x}_{i, j, k}}^{M}\left(t_{p}\right)-a_{\mathbf{x}_{i, j, k}}\left(t_{p+\frac{1}{2}}\right)}{\tau_{\mathbf{x}_{i, j, k}}\left(t_{p}\right)} \\
+
\end{gathered}
$$

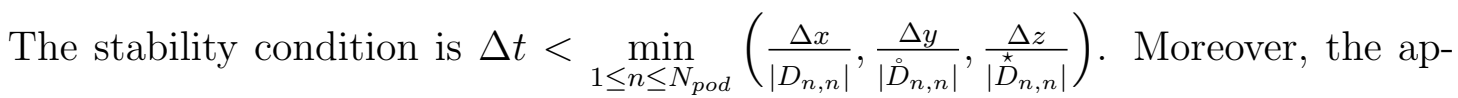
proximate density distribution function $\widetilde{f}$ is initialised with the approximate Maxwellian distribution function $\widetilde{M}_{f}$ corresponding to the initial density $\rho_{0}$, the initial macroscopic velocity $U_{0}$ and the initial temperature $T_{0}$ of the gas

$$
a_{\mathbf{x}_{i, j, k}}\left(t_{0}\right)=a_{\mathbf{x}_{i, j, k}}^{M}\left[\rho_{0}\left(\mathbf{x}_{i, j, k}\right), U_{0}\left(\mathbf{x}_{i, j, k}\right), T_{0}\left(\mathbf{x}_{i, j, k}\right)\right]
$$

where $a^{M}[\rho, U, T]$ are the coefficients of the approximate Maxwellian distribution function determined by $(\rho, U, T)$ (see section 3.2.2).

\subsection{Boundary conditions}

For the boundary conditions, ghost cells contain the approximate density distribution function $\widetilde{f}^{b c}$ determined by the boundary condition. In the following, $a^{b c}$ are the coefficients in the ghost cell which has an interface with the cell $\Omega_{\mathbf{x}}$ and $a$ are the coefficients in the cell $\Omega_{\mathbf{x}}$.

\subsubsection{Free flow}

For free flow, the approximate density distribution functions in the ghost cell $\mathbf{x}_{b c}$ and in the domain $\mathbf{x}$ are the same

$$
a^{b c}(t)=a(\mathbf{x}, t)
$$

In this case, the gradients are zeros at the boundary.

\subsubsection{Inflow/outflow}

The inflow (or outflow) is represented by a fluid state in the ghost cell $\mathbf{x}_{b c}$. The fluid state depends on the density $\rho_{b c}$, the macroscopic velocity $U_{b c}$ and the temperature $T_{b c}$, and corresponds to the approximate Maxwellian distribution function $\widetilde{M}_{f}$

$$
a^{b c}(t)=a^{M}\left[\rho_{b c}(t), U_{b c}(t), T_{b c}(t)\right]
$$




\subsubsection{Specular reflection}

The specular reflection is a wall reflecting the particles in opposite normal direction. The wall is moving with macroscopic velocity $U_{b c}$ and there is no mass and energy fluxes through the wall. The microscopic velocity of the particles becomes after the collision

$$
\xi_{r e f l}=\xi-2\left(\left(\xi-U_{b c}(t)\right) \cdot n_{w}\right) n_{w}
$$

where $n_{w}$ is the outward normal at the wall. The particles, hitting the wall, verify $0<\left(\xi-U_{b c}\right) \cdot n_{w}$ and the boundary condition reads

$$
\widehat{f}^{b c}(\xi, t)= \begin{cases}\widetilde{f}\left(\mathbf{x}, \xi_{\text {refl }}, t\right) & \text { if } 0<\left(\xi-U_{b c}(t)\right) \cdot n_{w} \\ \widetilde{f}(\mathbf{x}, \xi, t) & \text { otherwise }\end{cases}
$$

The function $\widehat{f}^{b c}$ do not necessarily belong to the subspace spanned by the basis functions $\Phi_{n}$. In the same way as in section 3.2.2, $\widehat{f}^{b c}$ is projected in order to conserve the moments $\overrightarrow{\rho_{b c}}$

$$
a^{b c}(t)=\Phi^{T} W \overrightarrow{f^{b c}}(t)+\Psi^{+}\left(\overrightarrow{\rho_{b c}}(t)-\Psi \Phi^{T} W \overrightarrow{f^{b c}}(t)\right)
$$

where $\overrightarrow{f^{b c}}(t)=\left(\widehat{f}^{b c}\left(\xi_{1}, t\right), \ldots, \widehat{f}^{b c}\left(\xi_{N_{\xi}}, t\right)\right)^{T}$ and $\overrightarrow{\rho_{b c}}(t)=\left\langle\widehat{f}^{b c}(\xi, t), m(\xi)\right\rangle_{\xi}$.

\subsubsection{Diffuse reflection}

The diffuse reflection is a wall reflecting the particles as Maxwellian distribution function. The wall has the macroscopic velocity $U_{b c}$, the temperature $T_{b c}$ and there is zero mass flux through the wall. In the ghost cell, the boundary condition is represented by the approximate Maxwellian distribution function determined by $\left(\rho_{b c}, U_{b c}, T_{b c}\right)$. The density $\rho_{b c}$ is computed to guarantee zero mass flux

$$
\begin{aligned}
& \int_{\left(\xi-U_{b c}(t)\right) \cdot n_{w}<0}\left(\xi-U_{b c}(t)\right) \widetilde{f}(\mathbf{x}, \xi, t) \mathrm{d} \xi+\int_{\left(\xi-U_{b c}(t)\right) \cdot n_{w}>0}\left(\xi-U_{b c}(t)\right) \widetilde{M}_{f}\left[\rho_{b c}(t), U_{b c}(t), T_{b c}(t)\right] \mathrm{d} \xi=0 \\
& \int_{\left(\xi-U_{b c}(t)\right) \cdot n_{w}<0}\left(\xi-U_{b c}(t)\right) \tilde{f}(\mathbf{x}, \xi, t) \mathrm{d} \xi+\int_{\left(\xi-U_{b c}(t)\right) \cdot n_{w}>0}\left(\xi-U_{b c}(t)\right) \rho_{b c}(t) \widetilde{M}_{f}\left[1, U_{b c}(t), T_{b c}(t)\right] \mathrm{d} \xi=0 \\
& \rho_{b c}(t)=-\frac{\left.\int_{\left(\xi-U_{b c}(t)\right) \cdot n_{w}<0}^{\left(\xi-U_{b c}\right.}(t)\right) \tilde{f}(\mathbf{x}, \xi, t) \mathrm{d} \xi}{\int_{\left(\xi-U_{b c}(t)\right) \cdot n_{w}>0}\left(\xi-U_{b c}(t)\right) \widetilde{M}_{f}\left[1, U_{b c}(t), T_{b c}(t)\right] \mathrm{d} \xi}
\end{aligned}
$$

where we use

$$
\begin{aligned}
\widetilde{M}_{f}[\rho, U, T] & =\Phi^{T} W M_{f}[\rho, U, T]+\Psi^{+}\left(\vec{\rho}[\rho, U, T]-\Psi \Phi^{T} W M_{f}[\rho, U, T]\right) \\
& =\Phi^{T} W \rho M_{f}[1, U, T]+\Psi^{+}\left(\rho \vec{\rho}[1, U, T]-\Psi \Phi^{T} W \rho M_{f}[1, U, T]\right) \\
& =\rho\left(\Phi^{T} W M_{f}[1, U, T]+\Psi^{+}\left(\vec{\rho}[1, U, T]-\Psi \Phi^{T} W M_{f}[1, U, T]\right)\right) \\
& =\rho \widetilde{M}_{f}[1, U, T]
\end{aligned}
$$


with $\vec{\rho}[\rho, U, T]$ the moments of $M_{f}[\rho, U, T]$. We reflect only the particles hitting the wall

$$
\widetilde{f}^{b c}(\xi, t)= \begin{cases}\rho_{b c}(t) \widetilde{M}_{f}\left[1, U_{b c}(t), T_{b c}(t)\right] & \text { if } 0<\left(\xi-U_{b c}(t)\right) \cdot n_{w} \\ \widetilde{f}(\mathbf{x}, \xi, t) & \text { otherwise }\end{cases}
$$

The coefficients $a_{n}^{b c}$ are given by projection

$$
a_{n}^{b c}(t)=\left\langle\widetilde{f}^{b c}(\xi, t), \Phi_{n}(\xi)\right\rangle_{\xi}
$$

This projection is exact and conserves the moments of $\widetilde{f}^{b c}$.

\section{Tests and results}

The reduced-order model is tested in 1D and 2D. We evaluate the accuracy and the run time of the reduced-order model with respect to the high-fidelity model. We use the approximation error

$$
\text { Error }=\frac{1}{2} \frac{\left\|\phi\left(\cdot, \cdot, t_{\max }\right)-\widetilde{\phi}\left(\cdot, \cdot, t_{\max }\right)\right\|_{L^{2}}}{\left\|\phi\left(\cdot, \cdot, t_{\max }\right)\right\|_{L^{2}}}+\frac{1}{2} \frac{\left\|\psi\left(\cdot, \cdot, t_{\max }\right)-\widetilde{\psi}\left(\cdot, \cdot, t_{\max }\right)\right\|_{L^{2}}}{\left\|\psi\left(\cdot, \cdot, t_{\max }\right)\right\|_{L^{2}}}
$$

and the run time

$$
\text { Run time }=\frac{\text { Reduced-order model run time }}{\text { High-fidelity model run time }}
$$

where $t_{\max }$ is the final time.

\subsection{Reconstruction tests}

In section 3.2.2 and 3.1.1.1, we propose to modify the approximate Maxwellian distribution function and the snapshot database in order to improve the reducedorder model. These modifications are evaluated on reconstruction tests where the density distribution function that we want to approximate is in the snapshot database used to built the basis functions. The prediction of density distribution function will be performed in the next section 5.2 .

\subsubsection{Reconstruction of a shock wave}

In the Galerkin method, the approximate Maxwellian distribution function $\widetilde{M}_{f}$ is the projection of the discrete Maxwellian distribution function $\bar{M}_{f}$ onto the basis functions $\Phi_{n}$. In section 3.2 .2 , we propose to compute the approximate Maxwellian distribution function $\widetilde{M}_{f}$ by constrained projection in order to conserve the mass, the momentum and the energy of the gas. We compare these two methods to compute the approximate Maxwellian distribution function $\widetilde{M}_{f}$. 
The test case is the Sod shock tube problem [30] with $K n=10^{-5}$. The physical space is $\Omega_{\mathbf{x}}=[0,1]$ discretized with $N_{x}=200$ points, the velocity space is $\Omega_{\xi}=[-10,10]$ discretized with $N_{\xi}=41$ points and the CFL condition is 0.1. The initial conditions are

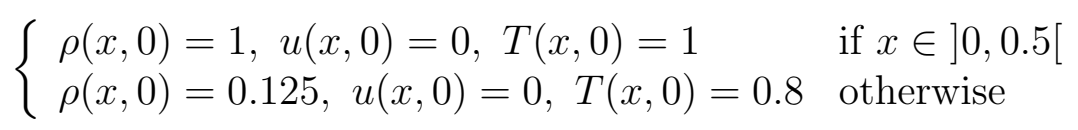

We consider free flow boundary conditions and the final time is $t_{\max }=0.12$. To build the basis functions, the database $S_{\phi}\left(\right.$ resp. $S_{\psi}$ ) contains snapshots of $\phi$ (resp. $\psi$ ) taken at any points in space and every 0.005 time units. The figure 4 shows the singular values of $\widehat{S}_{\phi}$ and $\widehat{S}_{\psi}$.

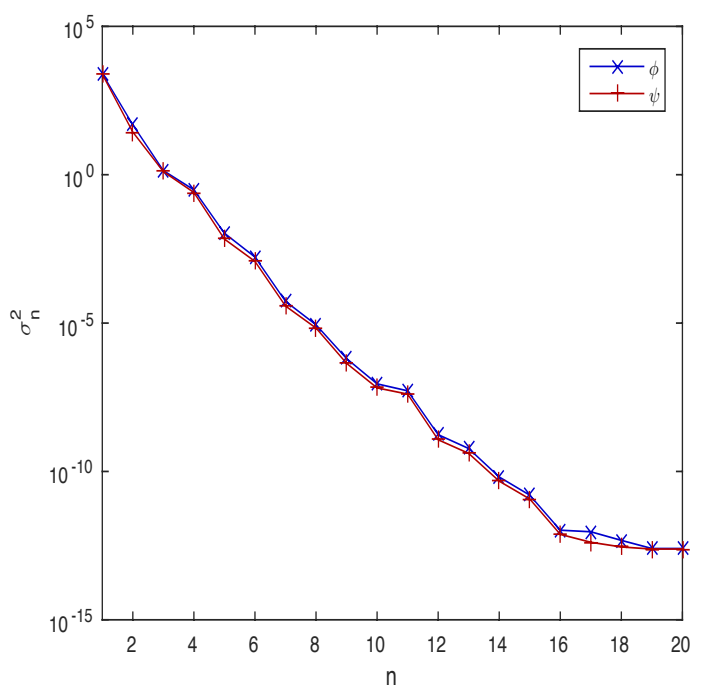

Figure 4: Singular values of $\widehat{S}_{\phi}$ (blue) and $\widehat{S}_{\psi}$ (red) for the shock wave reconstruction.

For $\Phi^{\phi}$ and $\Phi^{\psi}$, the singular values decrease quickly and the energy $E_{\text {pod }}$ captured by the basis functions $\Phi^{\phi}$ and $\Phi^{\psi}$ is more than $99.99 \%$ of the total energy with 3 basis functions (i.e. $7.3 \%$ of the complete basis). In Figure 5 , we plot the density $\rho$, the macroscopic velocity $u$, and the temperature $T$ of the gas at final time obtained by the reduced-order model where the approximate Maxwellian distribution function $\widetilde{M}_{f}$ is computed by constrained projection. 

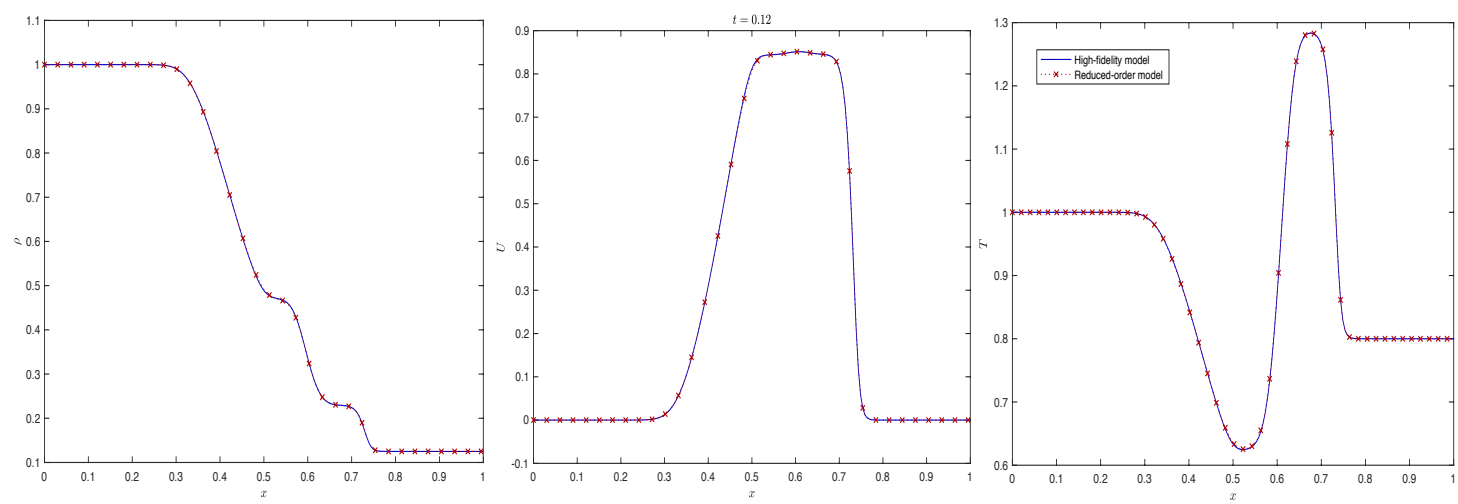

Figure 5: Density $\rho$, macroscopic velocity $u$ and temperature $T$ of the gas at final time for the shock wave reconstruction with $N_{\text {pod }}^{\phi}=N_{\text {pod }}^{\psi}=9$.

In Figure 6, we compare the approximation error and the run time of these two methods as a function of the number of basis functions $N_{\text {pod }}=N_{\text {pod }}^{\phi}=N_{\text {pod }}^{\psi}$.

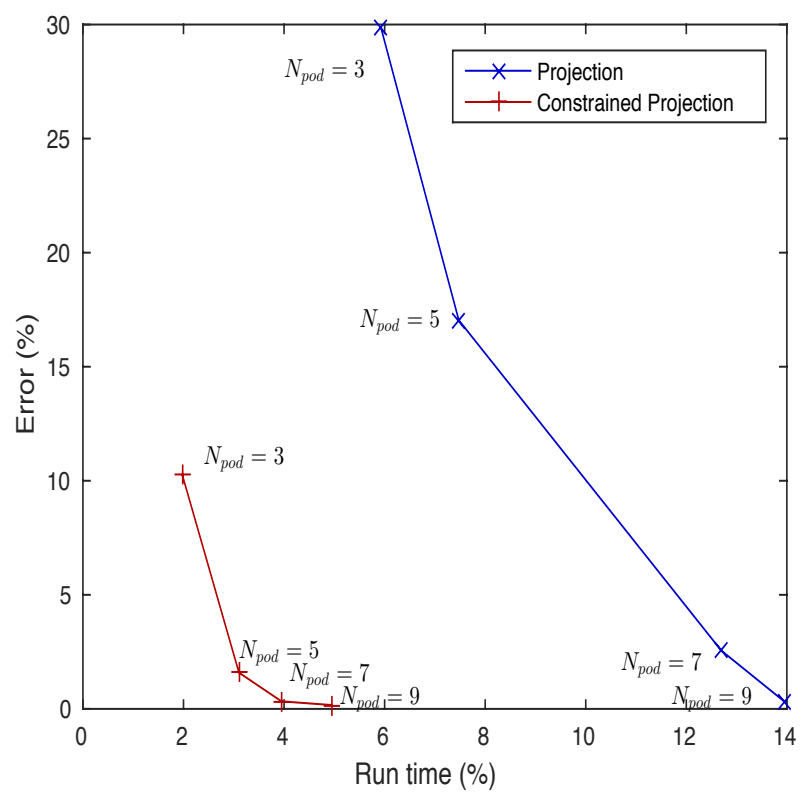

Figure 6: Comparison of the approximate Maxwellian distribution function $\widetilde{M}_{f}$.

The constrained projection leads to more accuracy because the approximate Maxwellian distribution function $\widetilde{M}_{f}$ verifies the equation (4). Moreover, the constrained projection is faster because we have an explicit formula for the coefficients while for the projection we have to solve a nonlinear system. More specifically in the projection, the Maxwellian distribution function $M_{f}$ must be computed to initialize the Newton-Raphson method (section 2.2). Then this nonlinear system is solved to obtain the discrete Maxwellian distribution function $\bar{M}_{f}$, which is finally projected onto the basis functions. In the constrained projection, the Maxwellian distribution function $M_{f}$ is computed and the formula (23) is used to obtain the 
approximate Maxwellian $\widetilde{M}_{f}$. Therefore, the approximate Maxwellian distribution function $\widetilde{M}_{f}$ is computed by constrained projection in the following since this approach is much faster and more accurate.

\subsubsection{Reconstruction of two boundary layers}

The second improvement of the reduced-oder model concerns the choice of the snapshots. Originally, the database $S$ contains snapshots of the density distribution functions $f$, because we want the basis functions $\Phi$ to be the best representation of $f$. In the section 3.2.2, the approximate Maxwellian distribution function $\widetilde{M}_{f}$ is represented by the basis functions $\Phi_{n}$. For this reason, we evaluate the interest of adding snapshots of the discrete Maxwellian distribution function $\bar{M}_{f}$.

We consider two walls (diffuse reflection) placed at $x=0$ and $x=1$ with different temperatures. The physical space is $\Omega_{\mathbf{x}}=[0,1]$ discretized with $N_{x}=100$ points, the velocity space is $\Omega_{\xi}=[-20,20]$ discretized with $N_{\xi}=100$ points and the final time is $t_{\max }=13.03$. The initial and boundary conditions are

$$
\left\{\begin{array}{l}
\rho_{0}(x)=1, u_{0}(x)=0, T_{0}(x)=1 \\
u_{b c}(0, t)=0, T_{b c}(0, t)=0.5, u_{b c}(1, t)=0, T_{b c}(1, t)=1.5
\end{array}\right.
$$

The CFL condition is 0.1 and the Knudsen number is $K n=10^{-2}$. To build the basis functions, we use two methods. In the first one, the database $S_{\phi}\left(\operatorname{resp} . S_{\psi}\right)$ contains snapshots of $\phi$ (resp. $\psi$ ) taken at any points in space and every 0.4 time units. In the second one, the database $S_{\phi}\left(\right.$ resp. $\left.S_{\psi}\right)$ contains snapshots of $\phi$ and $\bar{M}_{\phi}$ (resp. $\psi$ and $\bar{M}_{\psi}$ ) taken at any points in space and every 0.4 time units. Figure 7 shows the singular values of $\widehat{S}_{\phi}$ and $\widehat{S}_{\psi}$ for the two methods.
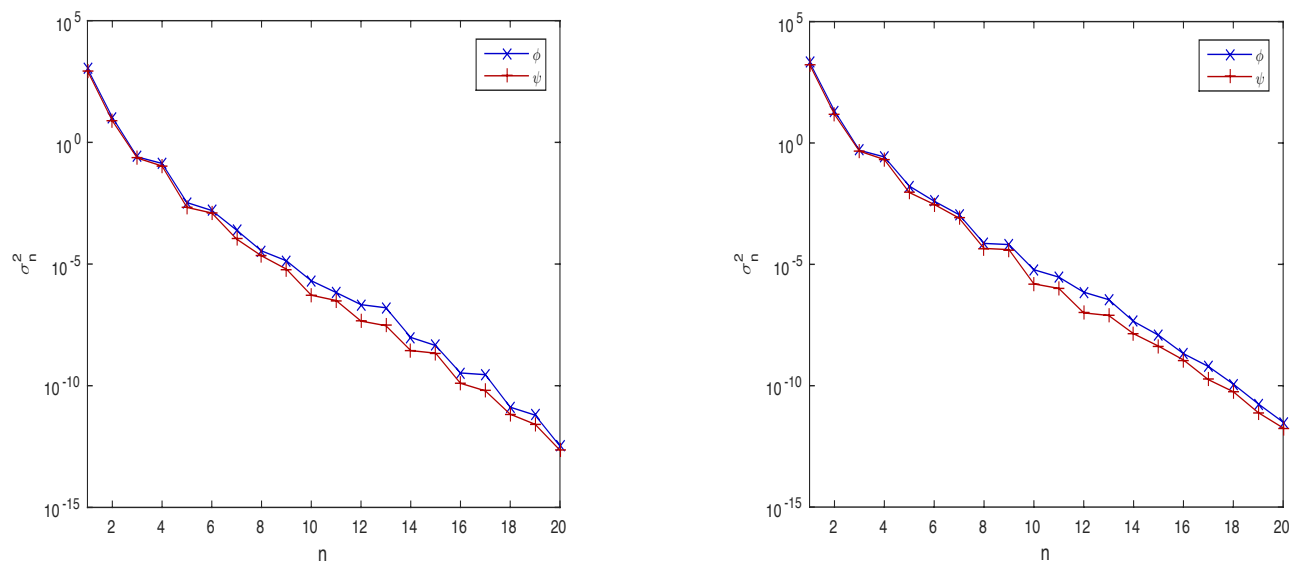

Figure 7: Singular values of $\widehat{S}_{\phi}$ and $\widehat{S}_{\psi}$ with (on the right) and without (on the left) Maxwellian snapshots for the boundary layers reconstruction.

The singular values of $\widehat{S}_{\phi}$ and $\widehat{S}_{\psi}$ decrease quickly and the energy $E_{\text {pod }}$ captured by the basis functions $\Phi^{\phi}$ and $\Phi^{\psi}$ is more than $99.99 \%$ of the total energy with 4 basis functions (i.e. $4 \%$ of the complete basis). In Figure 8, we plot the density $\rho$, the macroscopic velocity $u$ and the temperature $T$ of the gas at final time obtained 
by the reduced-order model where the database contains snapshots of the density and of the Maxwellian distribution functions.
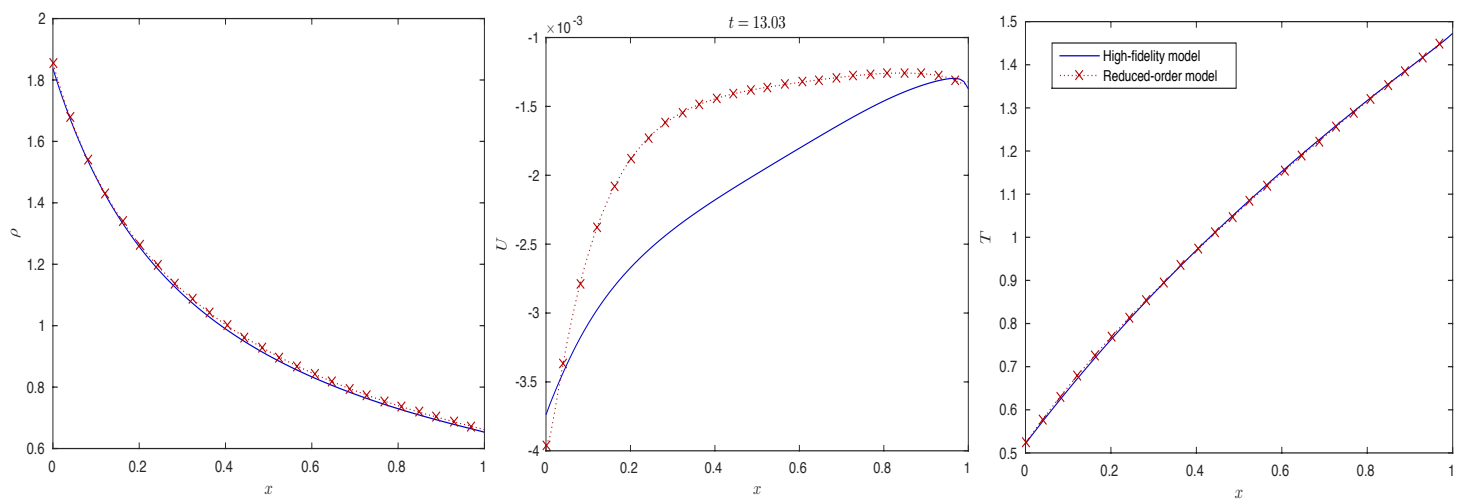

Figure 8: Density $\rho$, macroscopic velocity $u$ and temperature $T$ of the gas at final time for the boundary layers reconstruction with $N_{\text {pod }}^{\phi}=N_{\text {pod }}^{\psi}=12$.

In Figure 9, we compare the error and the run time of these two methods depending on the number of basis functions $N_{\text {pod }}=N_{\text {pod }}^{\phi}=N_{\text {pod }}^{\psi}$.

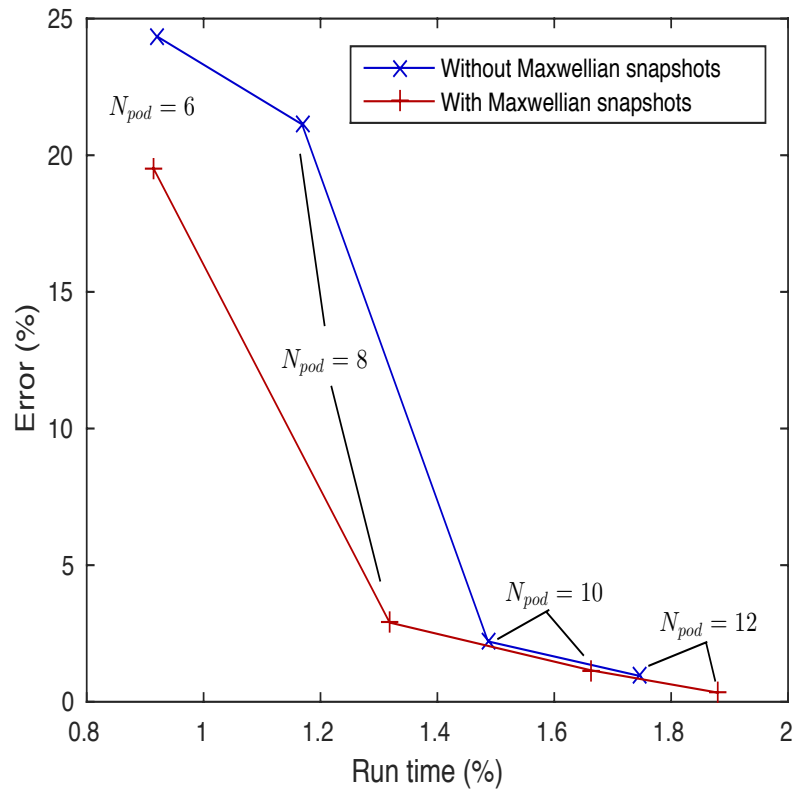

Figure 9: Comparison of the choice of the snapshot database $S$.

The enrichment of the snapshot database with the discrete Maxwellian distribution function reduces the error because the Maxwellian distribution function is better approximated. Moreover, it reduces the run time for $N_{\text {pod }}=6$ and increases it in the other cases. The run time is slightly different because the time step $\Delta t$ is determined by the basis functions (see section 4.1.3). A priori, we can 
not know which method leads to the biggest time step $\Delta t$ and in the following, the database contains snapshots of the density distribution function and of the discrete Maxwellian distribution function to increase the accuracy of the reducedorder model.

\subsubsection{Reconstruction of a vortex}

To evaluate the reduced-order model in $2 \mathrm{D}$, we consider a flow past a vertical plate [31]. The physical space is $\Omega_{\mathbf{x}}=[-1.33,2] \times[0,3.33]$ discretized with $N_{\mathbf{x}}=$ $64^{2}$ points and the velocity space is $\Omega_{\xi}=[-10,10]^{2}$ discretized with $N_{\xi}=41^{2}$ points. The final time is $t_{\max }=5.3332$ and the CFL condition is 0.5. An inflow condition is imposed at the boundary $(x=-1.33, x=2$ and $y=3.33)$ with the density $\rho_{b c}=1$, the macroscopic velocity $U_{b c}=(0.68,0)^{T}$ and the temperature $T_{b c}=1$. A vertical plate (specular reflection) is placed at $\left.\mathbf{x}=0 \times\right] 0,1[$. The initial and boundary conditions are

$$
\begin{cases}\rho(\mathbf{x}, 0)=1, u(\mathbf{x}, 0)=0.68, v(\mathbf{x}, 0)=0, T(\mathbf{x}, 0)=1 & \text { if } \mathbf{x} \in \Omega_{\mathbf{x}} \\ \rho(\mathbf{x}, t)=1, u(\mathbf{x}, t)=0.68, v(\mathbf{x}, t)=0, T(\mathbf{x}, t)=1 & \text { if } x=-1.33, t \in[0,5.33] \\ \rho(\mathbf{x}, t)=1, u(\mathbf{x}, t)=0.68, v(\mathbf{x}, t)=0, T(\mathbf{x}, t)=1 & \text { if } x=2, t \in[0,5.33] \\ \rho(\mathbf{x}, t)=1, u(\mathbf{x}, t)=0.68, v(\mathbf{x}, t)=0, T(\mathbf{x}, t)=1 & \text { if } y=3.33, t \in[0,5.33]\end{cases}
$$

and we consider a specular reflection at the boundary $y=0$. The basis functions $\Phi_{\phi}\left(\right.$ resp. $\left.\Phi_{\psi}\right)$ are built from the database $S_{\phi}$ (resp. $S_{\psi}$ ) containing snapshots of $\phi$ and $\bar{M}_{\phi}$ (resp. $\psi$ and $\bar{M}_{\psi}$ ) taken at any points in space and every 0.2665 time units. The Figure 10 shows the singular values of $\widehat{S}_{\phi}$ and $\widehat{S}_{\psi}$ at $K n=0.0345$.

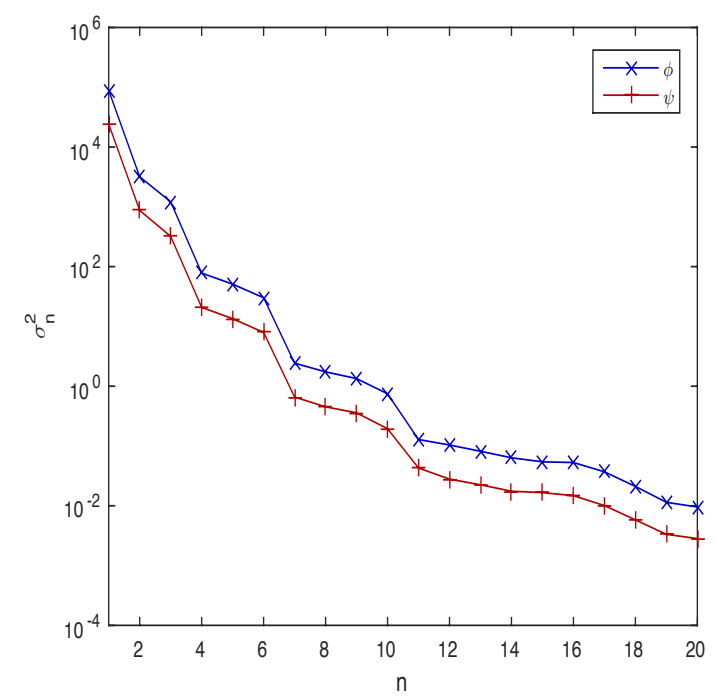

Figure 10: Singular values of $\widehat{S}_{\phi}$ (blue) and $\widehat{S}_{\psi}$ (red) for the vortex reconstruction.

The singular values of $\widehat{S}_{\phi}$ and $\widehat{S}_{\psi}$ decrease rapidly and the energy $E_{\text {pod }}$ captured by the basis functions $\Phi^{\phi}$ and $\Phi^{\psi}$ is more than $99.99 \%$ of the total energy with 6 basis functions (i.e. $0.4 \%$ of the complete basis). In Figure 11 , we plot the streamlines 
of the macroscopic velocity $U$ of the gas at final time obtained by the reduced-order model for different Knudsen number $K n \in\{0.0345,0.0689,0.115,0.23\}$.

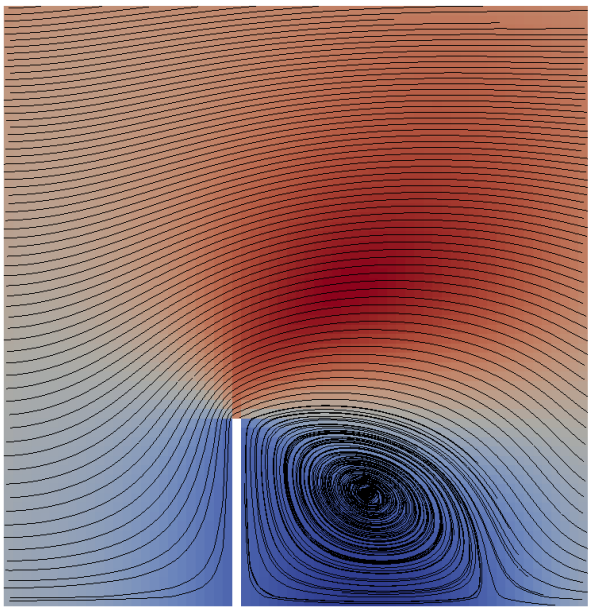

(a) $K n=0.0345$

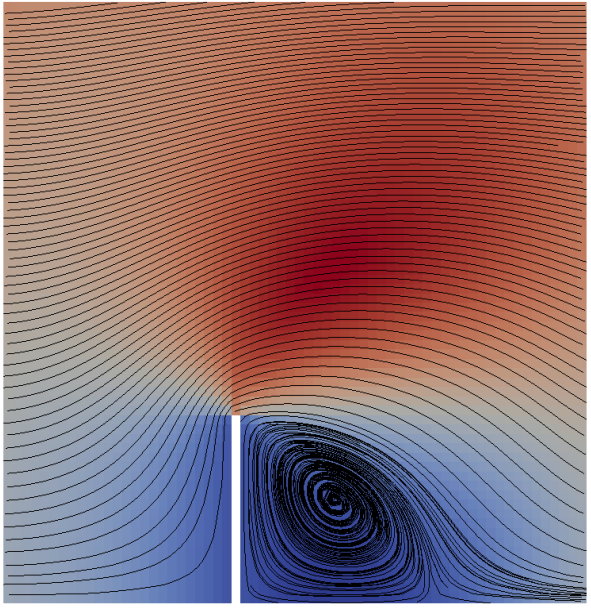

(c) $K n=0.115$

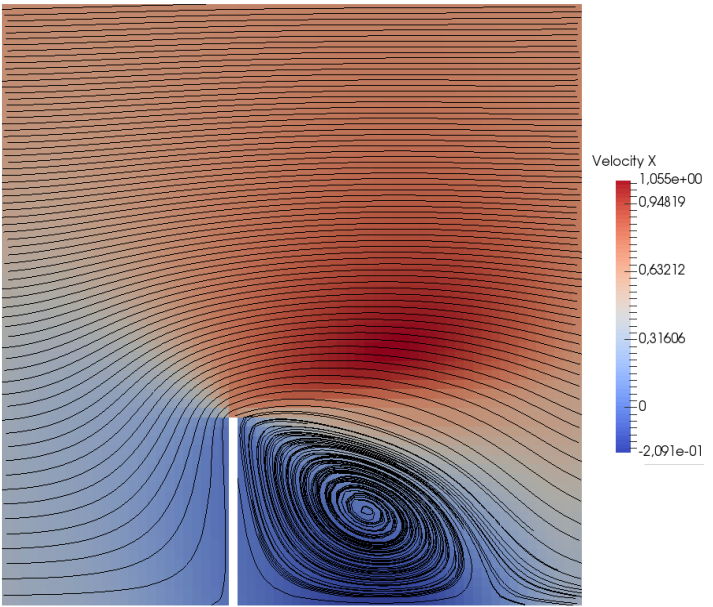

(b) $K n=0.0689$

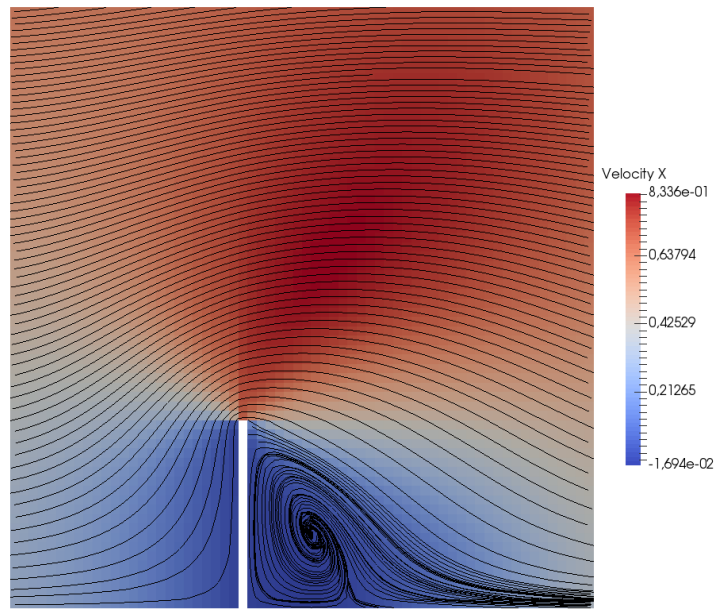

(d) $K n=0.23$

Figure 11: Streamlines of $U$ for the vortex reconstruction with $N_{\text {pod }}^{\phi}=N_{\text {pod }}^{\psi}=20$.

According to the high-fidelity simulations, a vortex is formed at the back of the wall and the vortex becomes stronger when the Knudsen number $K n$ decreases. In Figure 12, we evaluate the approximation error and the run time of the reducedorder model depending on the number of basis functions $N_{\text {pod }}=N_{\text {pod }}^{\phi}=N_{\text {pod }}^{\psi}$. 


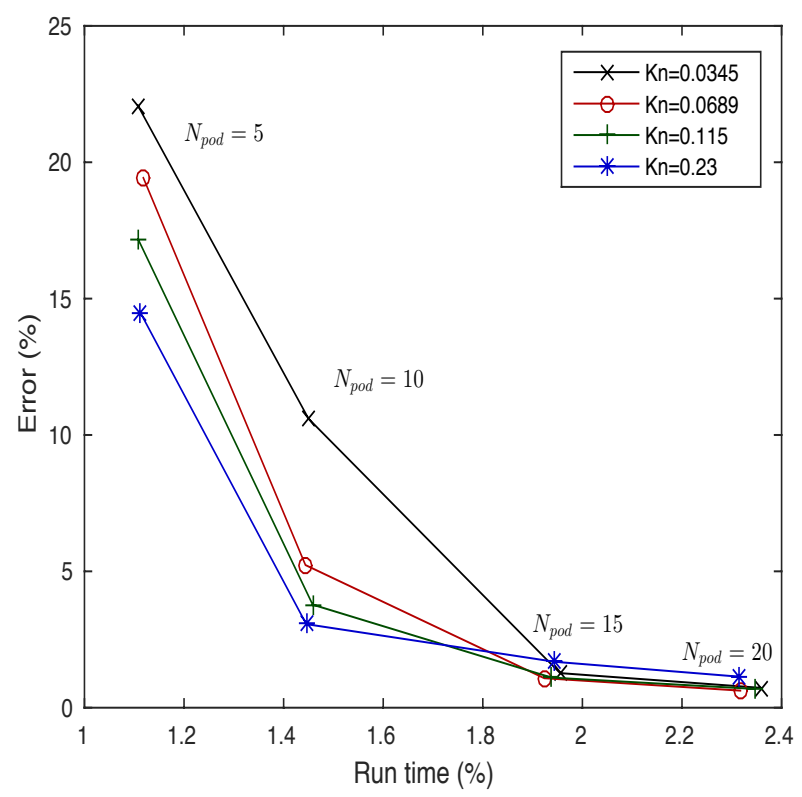

Figure 12: Results of the vortex reconstruction.

When the number of basis functions $N_{\text {pod }}$ increases, the approximation error decreases and the run time increases. The approximate density distribution function becomes more accurate because the subspace spanned by the basis functions converges to the total space. The computational cost increases because we solve more equations in the system $(19)$ and because the time step $\Delta t$ decreases. The number $N_{\text {pod }}$ of basis functions represents the trade-off between accuracy and computational cost. In average by taking $N_{\text {pod }}=20$ basis functions, the approximation error is less than $1 \%$ and the run time is divided by 45 approximately.

\subsection{Prediction tests}

In the previous tests, the density distribution function that we want to approximate was sampled with the high-fidelity model. We now predict density distribution functions which are not is in the snapshot database used to build the basis functions.

\subsubsection{Limits of the Proper Orthogonal Decomposition}

The difficulty of the prediction is that the basis functions are not necessarily the best representation in the least squares sense of the density distribution function that we want to predict. The density distribution function contained in the snapshots may be different from the density distribution function that we want to predict and the approximation error can be huge especially with few basis functions.

Given a set of snapshots $f_{l}^{\text {snap }}$, we begin to investigate which density distribution function $f^{\text {pred }}$ can be predicted. To simplify, we consider only one cell and one time step (i.e. the density distribution function that we want to predict $f^{p r e d}$ 
depends only on $\xi$ as the snapshots $f_{l}^{\text {snap }}$. Moreover by considering low Knudsen number, we assume that the density distribution functions $f^{\text {pred }}$ and $f_{l}^{\text {snap }}$ are Maxwellian distribution functions (see Figure 1) and are determined by the density $\rho$, the macroscopic velocity $U$ and the temperature $T$ of the gas.

The macroscopic velocity $U$ and the temperature $T$ are important for the prediction. If at least one of the snapshots (for example $f_{l^{*}}^{\text {snap }}$ ) has the same macroscopic velocity $U_{l^{*}}^{\text {snap }}=U^{\text {pred }}$ and the same temperature $T_{l^{*}}^{\text {snap }}=T^{\text {pred }}$ than the density distribution function $f^{\text {pred }}$, the basis functions $\Phi_{n}$ can give an accurate approximation of $f^{\text {pred }}$ by taking $N_{\text {pod }}$ large enough

$$
f^{\text {pred }}=\frac{\rho^{\text {pred }}}{\rho^{\text {snap }}} f^{\text {snap }} \approx \frac{\rho^{\text {pred }}}{\rho^{\text {snap }}} \sum_{n=1}^{N_{\text {pod }}} a_{n}^{\text {snap }} \Phi_{n} \approx \sum_{n=1}^{N_{\text {pod }}} \frac{\rho^{\text {pred }}}{\rho^{\text {snap }}} a_{n}^{\text {snap }} \Phi_{n} \approx \sum_{n=1}^{N_{\text {pod }}} a_{n}^{\text {pred }} \Phi_{n}
$$

More generally, every linear combinations of the snapshots can be approximated accurately.

Since the density distribution function decreases rapidly, we define $\operatorname{supp}(f) \subseteq$ $\mathbb{R}^{3}$ the subspace which contains at least $99.99 \%$ of the density distribution function $f$ (i.e. $\int_{\text {supp }(f)} f(\xi) \mathrm{d} \xi>99.99 \% \int_{\mathbb{R}^{3}} f(\xi) \mathrm{d} \xi$ ). In the case where $U_{l}^{\text {snap }} \neq U^{\text {pred }}$ or $T_{l}^{\text {snap }} \neq T^{\text {pred }}$ for all snapshots $f_{l}^{\text {snap }}$, the supports of the density distribution function $\operatorname{supp}\left(f^{\text {pred }}\right)$ and of the snapshots $\operatorname{supp}\left(f_{l}^{\text {snap }}\right)$ are different, which leads to approximation error. Moreover if $\operatorname{supp}\left(f^{\text {pred }}\right) \cap\left(\bigcup_{l} \operatorname{supp}\left(f_{l}^{\text {snap }}\right)\right)=\varnothing$, then the basis functions (associated to strictly positive singular values) are zero on supp (f pred) and can't represent $f^{\text {pred }}$.

Hence, to predict a distribution function $f^{\text {pred }}$, the database must contain snapshots of distribution functions corresponding to velocities $U_{l}^{\text {snap }} \approx U^{\text {pred }}$ and temperatures $T_{l}^{\text {snap }} \approx T^{\text {pred }}$. The snapshots are uniformly collected at all points in space and every time steps which provides a big database $\left(N_{\text {snaps }} \approx 10^{6}\right)$ with a large set of different velocities and temperatures. As explained before, additional low-fidelity snapshots can complete this sampling as shown in the following example.

\subsubsection{Prediction of a shock wave}

We consider the 1D case where the macroscopic velocities $u$ of the density distribution function that we want to approximate and of the density distribution function containing in the snapshot database are significantly different from each other.

The test case is the Sod shock tube problem with $K n=10^{-5}$. The physical space is $\Omega_{\mathbf{x}}=[0,1]$ discretized with $N_{x}=100$ points and the velocity space is $\Omega_{\xi}=[-20,20]$ discretized with $N_{\xi}=500$ points. We want to predict the density distribution function defined by

$$
\begin{cases}\rho(x, 0)=1, u(x, 0)=u^{0}, T(x, 0)=0.5 & \text { if } x \in] 0,0.5[ \\ \rho(x, 0)=0.125, u(x, 0)=u^{0}, T(x, 0)=0.4 & \text { otherwise }\end{cases}
$$


where $u^{0}$ belongs to $[-2,2]$, the boundary conditions are free flows and the final time is $t_{\text {max }}=0.1$. In this way, the density distribution functions depends only on the initial macroscopic velocity $u^{0}$. To build the basis functions, two high-fidelity simulations are available

$$
\begin{cases}\rho(x, 0)=1, u(x, 0)=-2, T(x, 0)=0.5 & \text { if } x \in] 0,0.5[ \\ \rho(x, 0)=0.125, u(x, 0)=-2, T(x, 0)=0.4 & \text { otherwise }\end{cases}
$$

and

$$
\begin{cases}\rho(x, 0)=1, u(x, 0)=2, T(x, 0)=0.5 & \text { if } x \in] 0,0.5[ \\ \rho(x, 0)=0.125, u(x, 0)=2, T(x, 0)=0.4 & \text { otherwise }\end{cases}
$$

with free flow boundary conditions and $t_{\max }=0.1$. The snapshot database $S_{\phi}$ (resp. $S_{\psi}$ ) contains snapshots of $\phi$ and $\bar{M}_{\phi}\left(\right.$ resp. $\psi$ and $\bar{M}_{\psi}$ ) of the simulations (S1) and (S2) taken at any point in space and every 0.005 time units. The temperature $T$ of the density distribution function of these two simulations are almost in the same interval $[0.38,0.6]$, but the macroscopic velocities $u$ are very different as shown by Figure 13 .
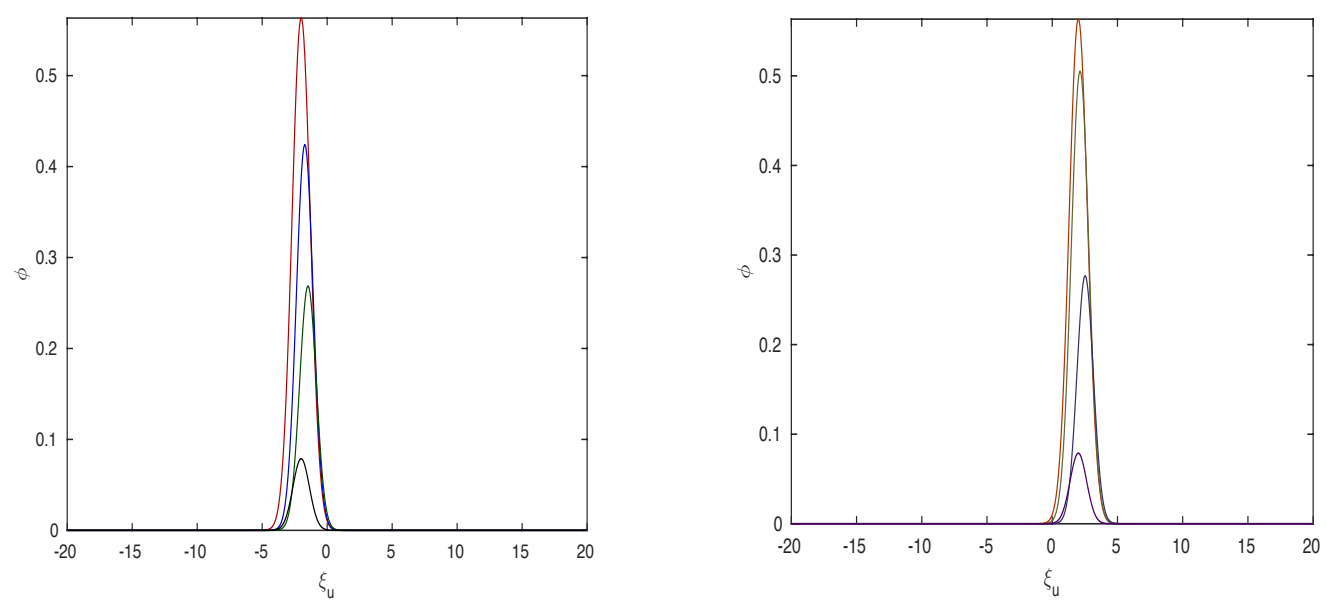

Figure 13: Examples of 4 snapshots of the simulation (S1) (left) and simulation (S2) (right).

The macroscopic velocities $u(x, t)=\int_{\mathbb{R}} \xi_{u} \phi\left(x, \xi_{u}, t\right) \mathrm{d} \xi_{u}$ correspond to the mean of the distribution function in velocity space and are around -2.5 (resp. 2.5) in the simulation (S1) (resp. (S2)). If we want to compute a density distribution function with macroscopic velocity 0 , the approximation error can be huge even with a large number of basis functions. Optimal transport can interpolate the density distribution function of the snapshots to have new density distribution function with velocities between -2.5 and 2.5. In our case, we use it to add new snapshots with macroscopic velocities around 0 to the database. At any point in space $x$ and every 0.005 time units, we compute the Wasserstein barycenter $s^{*}$ between the distribution function $s_{1}$ of the simulation (S1) and the distribution function $s_{2}$ of the simulation (S2) at barycentric coordinates $\left\{\left(s_{1}, \lambda_{1}=\frac{1}{2}\right),\left(s_{2}, \lambda_{2}=\frac{1}{2}\right)\right\}$. 

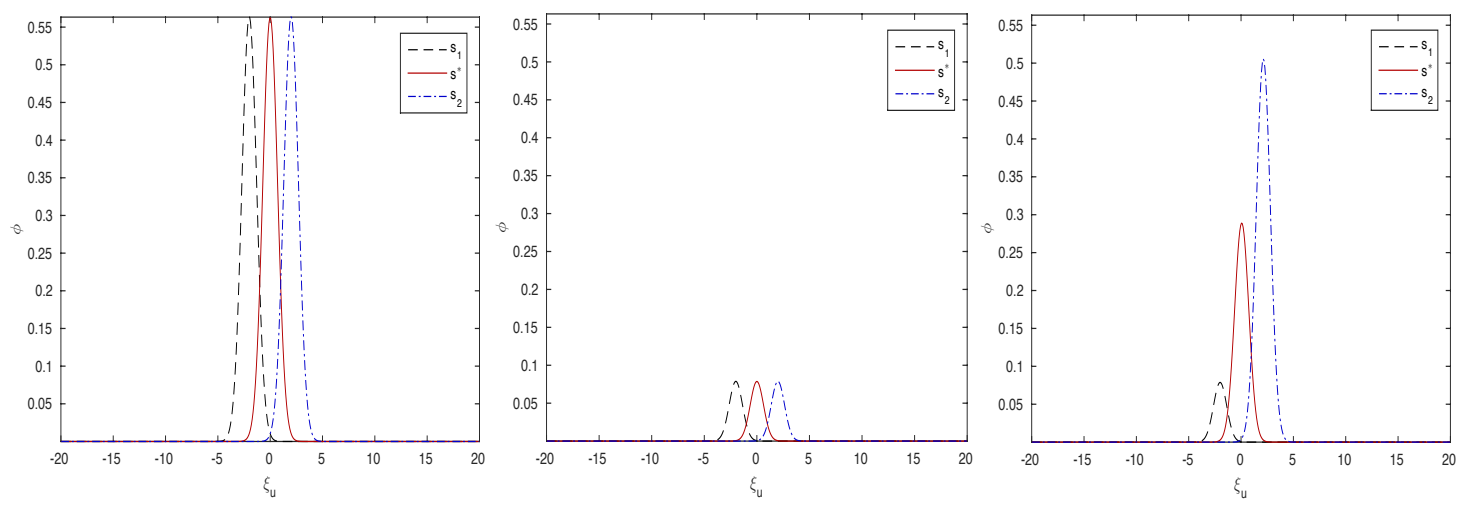

Figure 14: Examples of low-fidelity snapshots created from the simulations (S1) and (S2).

We evaluate two different basis functions $\Phi_{n}$. The first one is created from the snapshots of the simulations (S1) and (S2), and the second one is creates from the low-fidelity snapshots and from the snapshots of the simulations (S1) and (S2). In Figure 15, we evaluate the approximation error corresponding to different initial macroscopic velocities $u(x, 0)$.

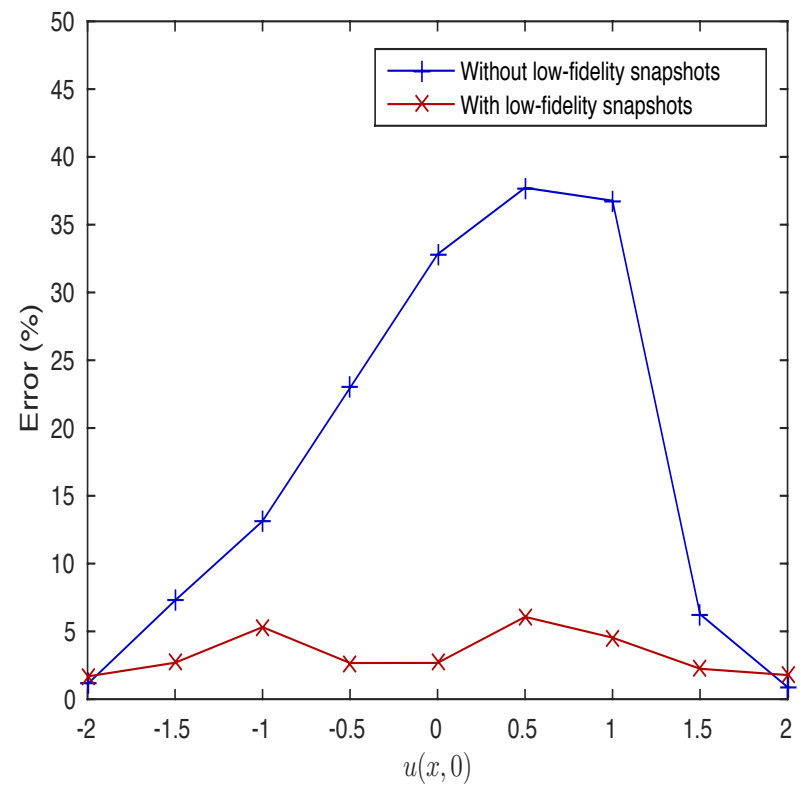

Figure 15: Results of the shock wave prediction with $N_{\text {pod }}^{\phi}=N_{\text {pod }}^{\psi}=9$.

The low-fidelity snapshots allow the basis functions to significantly improve the approximation of the density distribution functions corresponding to $u(x, 0) \in$ $[-1.5,1.5]$. For $u(x, 0)=-2$ and $u(x, 0)=2$, the approximation is slightly less accurate because the low-fidelity snapshots add useless information to represent the density distribution functions corresponding to $u(x, 0)=-2$ and $u(x, 0)=2$. In average, the reduced-order model is significantly more accurate with the lowfidelity snapshots. 


\subsubsection{Prediction of a vortex}

We consider now the case of a 2D flow past a vertical plate with $K n=0.0345$. The physical space is $\Omega_{\mathbf{x}}=[-1.33,2] \times[0,3.33]$ discretized with $N_{\mathbf{x}}=64^{2}$ points, the velocity space is $\Omega_{\xi}=[-10,10]^{2}$ discretized with $N_{\xi}=41^{2}$ points and the final time is $t_{\max }=5.3332$. We want to predict the density distribution functions with different velocities of the inflow $u^{b c}$ corresponding to Mach number in $[0.23,0.63]$

$$
\begin{cases}\rho(\mathbf{x}, 0)=1, u(\mathbf{x}, 0)=u^{b c}, v(\mathbf{x}, 0)=0, T(\mathbf{x}, 0)=1 & \text { if } \mathbf{x} \in \Omega_{\mathbf{x}} \\ \rho(\mathbf{x}, t)=1, u(\mathbf{x}, t)=u^{b c}, v(\mathbf{x}, t)=0, T(\mathbf{x}, t)=1 & \text { if } x=-1.33, t \in[0,5.33] \\ \rho(\mathbf{x}, t)=1, u(\mathbf{x}, t)=u^{b c}, v(\mathbf{x}, t)=0, T(\mathbf{x}, t)=1 & \text { if } x=2, t \in[0,5.33] \\ \rho(\mathbf{x}, t)=1, u(\mathbf{x}, t)=u^{b c}, v(\mathbf{x}, t)=0, T(\mathbf{x}, t)=1 & \text { if } y=3.33, t \in[0,5.33]\end{cases}
$$

where the boundary condition at $y=0$ is a specular reflection.

To build the snapshot database, we use only one high-fidelity simulation. The database $S_{\phi}$ (resp. $S_{\psi}$ ) contains snapshots of $\phi$ and $\bar{M}_{\phi}\left(\right.$ resp. $\psi$ and $\bar{M}_{\psi}$ ) corresponding to the simulation at Mach $=0.63$ taken at any points in space and every 0.2665 time units. In this way, the database contains all macroscopic velocities $U$ and temperatures $T$ which will appear in the simulations corresponding to $u^{b c}$ such that the Mach number is in $[0.23,0.63]$. For the macroscopic velocity $U$, the database contains distribution functions with the minimum (below the vortex) and the maximum (above the vortex) first component of macroscopic velocities $u$ which can arise as shown by Figure 16. Similarly, the simulation at Mach 0.63 contains the distribution functions with the minimum (at the right side of the plate) and the maximum (at the left side of the plate) temperatures $T$ which can appear.

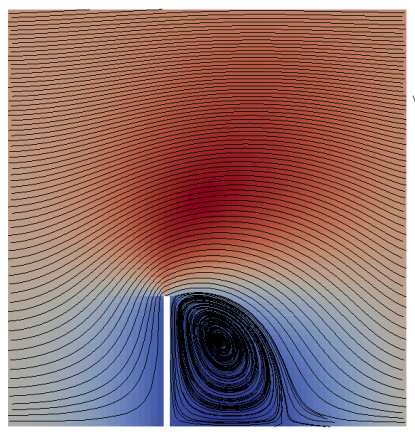

(a) Mach $=0.23$

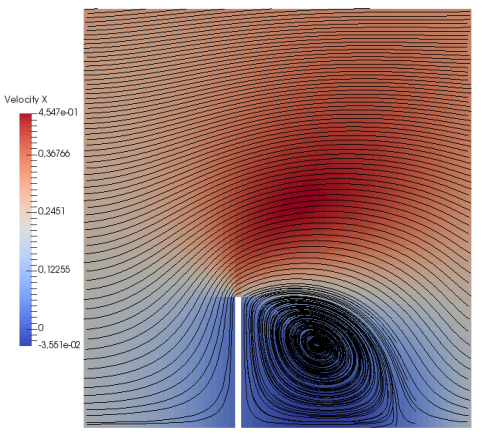

(b) Mach $=0.43$

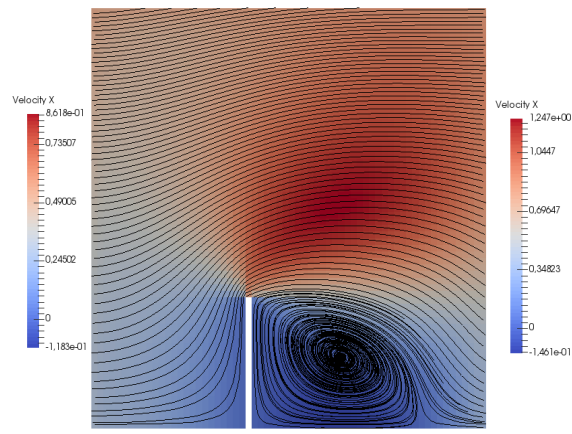

(c) Mach $=0.63$

Figure 16: Streamlines of $U$ for the vortex prediction with $N_{\text {pod }}^{\phi}=N_{\text {pod }}^{\psi}=20$.

In Figure 17, we evaluate the approximation error corresponding to different velocities of the inflow $u_{b c}$. 


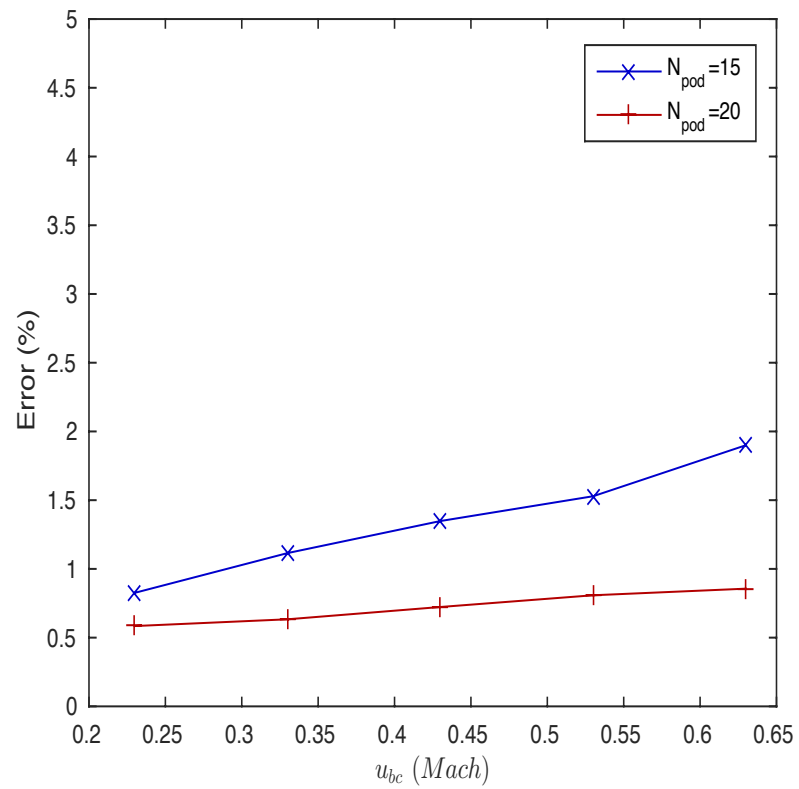

Figure 17: Results of the vortex prediction. Error as a function of the inlet velocity obtained for given Mach number.

For $u^{b c}$ corresponding to Mach in $[0.23,0.53]$, the basis functions are able to represent the density distribution function even if this one was not use to build the snapshot database. Moreover when the number of basis functions $N_{\text {pod }}=$ $N_{\text {pod }}^{\phi}=N_{\text {pod }}^{\psi}$ increases, the solution becomes more accurate. With $N_{\text {pod }}=20$ basis functions, the error is less than $1 \%$ for all prediction tests.

\section{Conclusion}

We have presented a reduced-order approximation of the BGK equation. In the reduced-order model, the density distribution function $f$ is represented in velocity space by few basis functions $\Phi_{n}$ so that the number of degrees of freedom is drastically reduced compared to the high-fidelity model. The basis functions $\Phi_{n}$ are built offline by POD and the approximate density distribution function $\widetilde{f}$ is computed online by the Galerkin method.

In the offline phase, we have proposed two improvements concerning the sampling of the high-fidelity model. The first one is to collect snapshots of $f$ and of the discrete Maxwellian $\bar{M}_{f}$ since the discrete Maxwellian distribution function $\bar{M}_{f}$ is to be approximated by the basis functions $\Phi_{n}$ in the reduced-order model. An additional improvement is to use optimal transport to complete the sampling of the high-fidelity model avoiding new simulations of the costly high-fidelity model. The new snapshots created by optimal transport add missing information and the basis functions are able to represent new features as shown in the shock wave prediction. 
In the online phase, we have shown that the system is hyperbolic by construction. We have presented a linear change of variables to solve by decoupling direction by direction the resulting partial differential equations in space and time. This system is then integrated by a first-order IMEX Runge-Kutta scheme in time and a first-order finite volume scheme in space. Also, we have shown how to compute the approximate Maxwellian distribution function $\widetilde{M}_{f}$ in order to conserve the mass, the momentum and the energy of the gas. This modification significantly improves the accuracy and reduces the computational cost of the reduced-order model.

The reduced-order model has been evaluated on $1 \mathrm{D}$ and $2 \mathrm{D}$ test cases. We have investigated the reconstruction and prediction of shock waves, boundary layers and vortices. The results demonstrate the accuracy of the reduced-order model (less than $1 \%$ of error) and a significant reduction of the run time (approximately divided by 45 ).

\section{Aknowledgements}

Experiments presented in this paper were carried out using the PlaFRIM experimental testbed, supported by Inria, CNRS (LABRI and IMB), Université de Bordeaux, Bordeaux INP and Conseil Régional d'Aquitaine (see https: //www.plafrim.fr//).

\section{References}

[1] P. L. Bhatnagar, E. P. Gross, M. Krook, A model for collision processes in gases. i. small amplitude processes in charged and neutral one-component systems, Physical review 94 (3) (1954) 511.

[2] L. Sirovich, Turbulence and the dynamics of coherent structures. i. coherent structures, Quarterly of applied mathematics 45 (3) (1987) 561-571.

[3] G. Berkooz, P. Holmes, J. L. Lumley, The proper orthogonal decomposition in the analysis of turbulent flows, Annual review of fluid mechanics 25 (1) (1993) 539-575.

[4] A. Iollo, S. Lanteri, J.-A. Désidéri, Stability properties of pod-galerkin approximations for the compressible navier-stokes equations, Theoretical and Computational Fluid Dynamics 13 (6) (2000) 377-396.

[5] K. Willcox, J. Peraire, Balanced model reduction via the proper orthogonal decomposition, AIAA journal 40 (11) (2002) 2323-2330.

[6] B. Galletti, C. Bruneau, L. Zannetti, A. Iollo, Low-order modelling of laminar flow regimes past a confined square cylinder, Journal of Fluid Mechanics 503 (2004) 161-170. 
[7] C. W. Rowley, T. Colonius, R. M. Murray, Model reduction for compressible flows using pod and galerkin projection, Physica D: Nonlinear Phenomena 189 (1-2) (2004) 115-129.

[8] C. W. Rowley, Model reduction for fluids, using balanced proper orthogonal decomposition, in: Modeling And Computations In Dynamical Systems: In Commemoration of the 100th Anniversary of the Birth of John von Neumann, World Scientific, 2006, pp. 301-317.

[9] M. Bergmann, C.-H. Bruneau, A. Iollo, Enablers for robust pod models, Journal of Computational Physics 228 (2) (2009) 516-538.

[10] G. Serre, P. Lafon, X. Gloerfelt, C. Bailly, Reliable reduced-order models for time-dependent linearized euler equations, Journal of Computational Physics 231 (15) (2012) 5176-5194.

[11] J. Östh, B. R. Noack, S. Krajnović, D. Barros, J. Borée, On the need for a nonlinear subscale turbulence term in pod models as exemplified for a highreynolds-number flow over an ahmed body, Journal of Fluid Mechanics 747 (2014) 518-544.

[12] R. Abgrall, D. Amsallem, R. Crisovan, Robust model reduction by $l^{\wedge}\{1\}$ 1 1-norm minimization and approximation via dictionaries: application to nonlinear hyperbolic problems, Advanced Modeling and Simulation in Engineering Sciences 3 (1) (2016) 1.

[13] F. Chinesta, A. Leygue, F. Bordeu, J. V. Aguado, E. Cueto, D. González, I. Alfaro, A. Ammar, A. Huerta, Pgd-based computational vademecum for efficient design, optimization and control, Archives of Computational Methods in Engineering 20 (1) (2013) 31-59.

[14] M. Barrault, Y. Maday, N. C. Nguyen, A. T. Patera, An empirical interpolation method: application to efficient reduced-basis discretization of partial differential equations, Comptes Rendus Mathematique 339 (9) (2004) 667672.

[15] S. Chaturantabut, D. C. Sorensen, Nonlinear model reduction via discrete empirical interpolation, SIAM Journal on Scientific Computing 32 (5) (2010) $2737-2764$.

[16] U. M. Ascher, S. J. Ruuth, R. J. Spiteri, Implicit-explicit runge-kutta methods for time-dependent partial differential equations, Applied Numerical Mathematics 25 (2-3) (1997) 151-167.

[17] K. Christopher A, C. Mark H, Additive runge-kutta schemes for convectiondiffusion-reaction equations. 
[18] L. Pareschi, G. Russo, Implicit-explicit runge-kutta schemes and applications to hyperbolic systems with relaxation, Journal of Scientific computing 25 (1) (2005) 129-155.

[19] H. K. Versteeg, W. Malalasekera, An introduction to computational fluid dynamics: the finite volume method, Pearson Education, 2007.

[20] F. Bernard, Efficient asymptotic preserving schemes for bgk and es-bgk models on cartesian grids, http://porto.polito.it/2617451/1/PhD_thesis. $\operatorname{pdf}(2015)$.

[21] L. Mieussens, Discrete-velocity models and numerical schemes for the boltzmann-bgk equation in plane and axisymmetric geometries, Journal of Computational Physics 162 (2) (2000) 429-466.

[22] H. Cabannes, R. Gatignol, L. Luol, The discrete boltzmann equation, Lecture Notes at University of California, Berkley (1980) 1-65.

[23] C. Chu, Kinetic-theoretic description of the formation of a shock wave, The Physics of Fluids 8 (1) (1965) 12-22.

[24] G. Monge, Mémoire sur la théorie des déblais et des remblais, Histoire de l'Académie Royale des Sciences de Paris.

[25] J. Solomon, F. De Goes, G. Peyré, M. Cuturi, A. Butscher, A. Nguyen, T. Du, L. Guibas, Convolutional wasserstein distances: Efficient optimal transportation on geometric domains, ACM Transactions on Graphics (TOG) 34 (4) (2015) 66.

[26] J.-D. Benamou, G. Carlier, M. Cuturi, L. Nenna, G. Peyré, Iterative bregman projections for regularized transportation problems, SIAM Journal on Scientific Computing 37 (2) (2015) A1111-A1138.

[27] G. Forsythe, M. Malcolm, C. Moler, Computer methods for mathematical computation prentice-hall, Englewood Cliffs, New Jersey.

[28] M. Cuturi, Sinkhorn distances: Lightspeed computation of optimal transport, in: Advances in neural information processing systems, 2013, pp. 2292-2300.

[29] M. Cuturi, A. Doucet, Fast computation of wasserstein barycenters, in: International Conference on Machine Learning, 2014, pp. 685-693.

[30] G. A. Sod, A survey of several finite difference methods for systems of nonlinear hyperbolic conservation laws, Journal of computational physics 27 (1) (1978) 1-31.

[31] G. Bird, Molecular gas dynamics and the direct simulation monte carlo of gas flows, Clarendon, Oxford 508 (1994) 128. 